\title{
Classical Particle in Presence of Magnetic Field, Hyperbolic Lobachevsky and Spherical Riemann Models`
}

\author{
V.V. KUDRYASHOV, Yu.A. KUROCHKIN, E.M. OVSIYUK and V.M. RED'KOV \\ Institute of Physics, National Academy of Sciences of Belarus, Minsk, Belarus \\ E-mail:kudryash@dragon.bas-net.by,y.kurochkin@ifanbel.bas-net.by, \\ mozlena@tut.by,redkov@dragon.bas-net.by
}

Received July 20, 2009, in final form December 29, 2009; Published online January 10, 2010 doi:10.3842/SIGMA.2010.004

\begin{abstract}
Motion of a classical particle in 3-dimensional Lobachevsky and Riemann spaces is studied in the presence of an external magnetic field which is analogous to a constant uniform magnetic field in Euclidean space. In both cases three integrals of motions are constructed and equations of motion are solved exactly in the special cylindrical coordinates on the base of the method of separation of variables. In Lobachevsky space there exist trajectories of two types, finite and infinite in radial variable, in Riemann space all motions are finite and periodical. The invariance of the uniform magnetic field in tensor description and gauge invariance of corresponding 4-potential description is demonstrated explicitly. The role of the symmetry is clarified in classification of all possible solutions, based on the geometric symmetry group, $S O(3,1)$ and $S O(4)$ respectively.
\end{abstract}

Key words: Lobachevsky and Riemann spaces; magnetic field; mechanics in curved space; geometric and gauge symmetry; dynamical systems

2010 Mathematics Subject Classification: 37J35; 70G60; 70H06; 74H05

\section{Introduction}

In the paper exact solutions for classical problem of a particle in a magnetic field on the background of hyperbolic Lobachevsky $H_{3}$ and spherical Riemann $S_{3}$ space models will be constructed explicitly.

The grounds to examine these problems are as follows: these both are extensions for a wellknown problem in theoretical physics - a particle in a uniform magnetic field [15]; they can be used to describe behavior of charged particles in macroscopic magnetic field in the context of astrophysics. The form of the magnetic field in the models $H_{3}$ and $S_{3}$ was introduced earlier in $[2,3,4]$ where the quantum-mechanical variant (for the Shrödinger equation) of the problem had been solved as well and generalized formulas for Landau levels $[14,15,16]$ had been produced. A part of results of the paper was presented in the talk given in [13].

Previously, the main attention was given to Landau problem in 2-dimensional case: many important mathematical and physical results were obtained, see in $[1,5,6,8,9,12,17,19]$. Comprehensive discussion of the general problem of integrability of classical and quantum systems in Lobachevsky and Riemann $3 \mathrm{D}$ and $2 \mathrm{~A}$ models see in $[7,10,11]$ and references therein. It is known that $2 \mathrm{D}$ and $3 \mathrm{D}$ systems exhibit properties which are radically different. Our treatment will concern only a 3-dimensional case.

${ }^{\star}$ This paper is a contribution to the Proceedings of the Eighth International Conference "Symmetry in Nonlinear Mathematical Physics" (June 21-27, 2009, Kyiv, Ukraine). The full collection is available at http://www.emis.de/journals/SIGMA/symmetry2009.html 


\section{Newton second law in Lobachevsky space}

Motion of a classical particle in external electromagnetic and gravitational fields is described by the known equation [15]

$$
m c^{2}\left(\frac{d^{2} x^{\alpha}}{d s^{2}}+\Gamma_{\beta \sigma}^{\alpha} \frac{d x^{\beta}}{d s} \frac{d x^{\sigma}}{d s}\right)=e F^{\alpha \rho} U_{\rho}
$$

where Christoffel symbols are determined by metrical structure of a space-time (the signature +--- is used). In (2.1) it is useful to perform $(3+1)$-splitting

$$
\begin{aligned}
& m c^{2} \frac{d^{2} x^{0}}{d s^{2}}=e\left(F^{01} U_{1}+F^{02} U_{2}+F^{03} U_{3}\right), \\
& m c^{2}\left(\frac{d^{2} x^{1}}{d s^{2}}+\Gamma_{j k}^{1} \frac{d x^{j}}{d s} \frac{d x^{k}}{d s}\right)=e F^{10} U_{0}+e F^{12} U_{2}+e F^{13} U_{3}, \\
& m c^{2}\left(\frac{d^{2} x^{2}}{d s^{2}}+\Gamma^{2}{ }_{j k} \frac{d x^{j}}{d s} \frac{d x^{k}}{d s}\right)=e F^{20} U_{0}+e F^{21} U_{1}+e F^{23} U_{3}, \\
& m c^{2}\left(\frac{d^{2} x^{3}}{d s^{2}}+\Gamma_{j k}^{3} \frac{d x^{j}}{d s} \frac{d x^{k}}{d s}\right)=e F^{30} U_{0}+e F^{31} U_{1}+e F^{32} U_{2} .
\end{aligned}
$$

In (2.2)-(2.5) usual SI units are used, so the Christoffel symbols $\Gamma_{j k}^{i}$ are measured in (meter) ${ }^{-1}$. With conventional notation [15]

$$
\begin{aligned}
& \left(F^{\alpha \beta}\right)=\left|\begin{array}{rrrr}
0 & -E^{1} & -E^{2} & -E^{3} \\
E^{1} & 0 & -c B^{3} & c B^{2} \\
E^{2} & -c B^{3} & 0 & -c B^{1} \\
E^{3} & -c B^{2} & c B^{1} & 0
\end{array}\right|, \\
& U^{\alpha}=\frac{d t}{d s} \frac{d}{d t}\left(d x^{0}, d x^{i}\right)=\frac{1}{\sqrt{1-V^{2} / c^{2}}}\left(1, \frac{V^{i}}{c}\right), \\
& V^{i}=\frac{d x^{i}}{d t}, \quad V^{2}=-g_{k i}(x) V^{k} V^{i}, \quad \frac{d x^{0}}{d s}=\left(\frac{c d t \sqrt{1-V^{2} / c^{2}}}{c d t}\right)^{-1}=\frac{1}{\sqrt{1-V^{2} / c^{2}}}
\end{aligned}
$$

equations (2.2)-(2.5) give

$$
\begin{aligned}
& \frac{d}{d t}\left(\frac{m c^{2}}{\sqrt{1-V^{2} / c^{2}}}\right)=e\left[-g_{i k}(x) E^{i} V^{k}\right], \\
& \frac{d}{d t} \frac{V^{1}}{\sqrt{1-V^{2} / c^{2}}}+\frac{1}{\sqrt{1-V^{2} / c^{2}}} \Gamma_{j k}^{1} V^{j} V^{k}=\frac{e}{m} E^{1}-\frac{e}{m}\left(V_{2} B^{3}-V_{3} B^{2}\right), \\
& \frac{d}{d t} \frac{V^{2}}{\sqrt{1-V^{2} / c^{2}}}+\frac{1}{\sqrt{1-V^{2} / c^{2}}} \Gamma_{j k}^{2} V^{j} V^{k}=\frac{e}{m} E^{2}-\frac{e}{m}\left(V_{3} B^{1}-V_{1} B^{3}\right), \\
& \frac{d}{d t} \frac{V^{3}}{\sqrt{1-V^{2} / c^{2}}}+\frac{1}{\sqrt{1-V^{2} / c^{2}}} \Gamma_{j k}^{3} V^{j} V^{k}=\frac{e}{m} E^{3}-\frac{e}{m}\left(V_{1} B^{2}-V_{2} B^{1}\right) .
\end{aligned}
$$

Firstly, we will be interested in non-relativistic case $^{1}$, when all equations become simpler

$$
\frac{d}{d t} \frac{m V^{2}}{2}=e\left(-g_{i k} E^{i} V^{k}\right)
$$

\footnotetext{
${ }^{1}$ Extension to the relativistic case will be performed in the end of the paper.
} 


$$
\begin{aligned}
\frac{d}{d t} V^{1}+\Gamma_{j k}^{1} V^{j} V^{k} & =\frac{e}{m} E^{1}-\frac{e}{m}\left(V_{2} B^{3}-V_{3} B^{2}\right), \\
\frac{d}{d t} V^{2}+\Gamma_{j k}^{2} V^{j} V^{k} & =\frac{e}{m} E^{2}-\frac{e}{m}\left(V_{3} B^{1}-V_{1} B^{3}\right), \\
\frac{d}{d t} V^{3}+\Gamma_{j k}^{3}{ }_{j} V^{j} V^{k} & =\frac{e}{m} E^{3}-\frac{e}{m}\left(V_{1} B^{2}-V_{2} B^{1}\right) .
\end{aligned}
$$

\section{Particle in a uniform magnetic field, hyperbolic model $\boldsymbol{H}_{3}$}

Let us start with the known 4-vector potential of a uniform magnetic field in flat space [15]

$$
\mathbf{A}=\frac{1}{2} c \mathbf{B} \times \mathbf{r}, \quad \mathbf{B}=(0,0, B), \quad \frac{A^{a}}{c}=\frac{B}{2}(0 ;-r \sin \phi, r \cos \phi, 0) .
$$

From (3.1), after transformation to cylindric coordinates we obtain

$$
A_{t}=0, \quad A_{r}=0, \quad A_{z}=0, \quad A_{\phi}=-\frac{c B r^{2}}{2} .
$$

The only non-vanishing constituent of the electromagnetic tensor reads

$$
F_{\phi r}=\partial_{\phi} A_{r}-\partial_{r} A_{\phi}=c B r
$$

which satisfies the Maxwell equations

$$
\frac{1}{\sqrt{-g}} \frac{\partial}{\partial x^{\alpha}} \sqrt{-g} F^{\alpha \beta}=0 \Longrightarrow \frac{1}{r} \frac{\partial}{\partial r} r F^{\phi r}=\frac{1}{r} \frac{\partial}{\partial r} r\left(\frac{1}{r^{2}}\right) c B r \equiv 0 .
$$

Now we are to extend the concept of a uniform magnetic field to the Lobachevsky model $H_{3}$. Thirty four orthogonal coordinate systems in this space were found by Olevsky [18]. An idea is to select among them some curved analogue for cylindric coordinates and determine with their help an appropriate solution to Maxwell equations in Lobachevsky space. In [18], under the number XI we see the following coordinates

$$
\begin{aligned}
& d S^{2}=c^{2} d t^{2}-\rho^{2}\left[\cosh ^{2} z\left(d r^{2}+\sinh ^{2} r d \phi^{2}\right)+d z^{2}\right], \\
& z \in(-\infty,+\infty), \quad r \in[0,+\infty), \quad \phi \in[0,2 \pi], \\
& u_{1}=\cosh z \sinh r \cos \phi, \quad u_{2}=\cosh z \sinh r \sin \phi, \\
& u_{3}=\sinh z, \quad u_{0}=\cosh z \cosh r, \\
& u_{0}^{2}-u_{1}^{2}-u_{2}^{2}-u_{3}^{3}=1, \quad u_{0}=+\sqrt{1+\mathbf{u}^{2}},
\end{aligned}
$$

the curvature radius $\rho$ is taken as a unit length. In the limit $\rho \rightarrow \infty$ the coordinates (3.2) reduce to ordinary cylindric ones in the flat space. By definition, the uniform magnetic field in the Lobachevsky space is given by 4-potential of the form

$$
A_{\phi}=-2 c B \rho^{2} \sinh ^{2} \frac{r}{2}=-c B \rho^{2}(\cosh r-1) .
$$

It behaves properly in the limit $\rho \longrightarrow \infty$, besides it corresponds to an electromagnetic tensor which evidently satisfies the Maxwell equations in $H_{3}$

$$
\begin{aligned}
& F_{\phi r}=-\frac{1}{\rho} \partial_{r} A_{\phi}=c B \rho \sinh r \\
& \frac{1}{\cosh ^{2} z \sinh r} \frac{\partial}{\partial r} \cosh ^{2} z \sinh r\left(\frac{1}{\cosh ^{4} z \sinh ^{2} r}\right) B \sinh r \equiv 0 .
\end{aligned}
$$


In the absence of an electric field, the non-relativistic equations (2.7)-(2.9) read

$$
\begin{aligned}
\frac{d V^{r}}{d t}+\Gamma^{r}{ }_{j k} V^{j} V^{k} & =\frac{e}{m} F^{r \phi} V_{\phi}, \\
\frac{d V^{\phi}}{d t}+\Gamma^{\phi}{ }_{j k} V^{j} V^{k} & =\frac{e}{m} F^{\phi r} V_{r}, \\
\frac{d V^{z}}{d t}+\Gamma^{z}{ }_{j k} V^{j} V^{k} & =0,
\end{aligned}
$$

where the Christoffel symbols are

$$
\begin{aligned}
& \Gamma_{j k}^{r}=\left|\begin{array}{ccc}
0 & 0 & \tanh z \\
0 & -\sinh r \cosh r & 0 \\
\tanh z & 0 & 0
\end{array}\right|, \quad \Gamma_{j k}^{\phi}=\left|\begin{array}{ccc}
0 & \operatorname{coth} r & 0 \\
\operatorname{coth} r & 0 & \tanh z \\
0 & \tanh z & 0
\end{array}\right|, \\
& \Gamma^{z}{ }_{j k}=\left|\begin{array}{ccc}
-\cosh z \sinh z & 0 & 0 \\
0 & -\sinh z \cosh z \sinh ^{2} r & 0 \\
0 & 0 & 0
\end{array}\right| .
\end{aligned}
$$

It makes sense to recall a similar problem in flat space, here the Christoffel symbols are simpler

$$
\Gamma_{j k}^{r}=\left|\begin{array}{ccc}
0 & 0 & 0 \\
0 & -r & 0 \\
0 & 0 & 0
\end{array}\right|, \quad \Gamma_{j k}^{\phi}=\left|\begin{array}{ccc}
0 & r^{-1} & 0 \\
r^{-1} & 0 & 0 \\
0 & 0 & 0
\end{array}\right|, \quad \Gamma_{j k}^{z}=0
$$

and equations of motion read in space $E_{3}$,

$$
\frac{d V^{r}}{d t}-r V^{\phi} V^{\phi}=\frac{e}{m} B r V^{\phi}, \quad \frac{d V^{\phi}}{d t}+\frac{2}{r} V^{r} V^{\phi}=-q \frac{B}{r} V^{r}, \quad \frac{d V^{z}}{d t}=0 .
$$

In fact, a simplest solution of these equations is well-known: the particle moves on a cylindric surface oriented along the axis $z$ according to the law $\phi(t)=\omega t+\phi_{0}$, correspondingly equations (3.4) take the form

$$
\frac{d^{2} r}{d t^{2}}=r \omega\left(\omega+\frac{e}{m} B\right), \quad \frac{d r}{d t} \frac{1}{r}\left(2 \omega+\frac{e}{m} B\right)=0 \Longrightarrow \frac{d r}{d t}=0,
$$

and the simplest solution is given by

$$
\omega=-\frac{e B}{m}, \quad \phi(t)=\omega t+\phi_{0}, \quad r(t)=r_{0}, \quad z(t)=z(t)=z_{0}+V_{0}^{z} t .
$$

Now, let us turn to the problem in Lobachevsky model - equations (3.3) give

$$
\begin{aligned}
& \frac{d V^{r}}{d t}+2 \tanh z V^{r} V^{z}-\sinh r \cosh r V^{\phi} V^{\phi}=B \frac{\sinh r}{\cosh ^{2} z} V^{\phi}, \\
& \frac{d V^{\phi}}{d t}+2 \operatorname{coth} r V^{\phi} V^{r}+2 \tanh z V^{\phi} V^{z}=-B \frac{1}{\cosh ^{2} z \sinh r} V^{r}, \\
& \frac{d V^{z}}{d t}-\sinh z \cosh z V^{r} V^{r}-\sinh z \cosh z \sinh ^{2} r V^{\phi} V^{\phi}=0 .
\end{aligned}
$$

It should be stressed that in (3.5) all quantities (coordinates $t, r, \phi, z$ as well ) are dimensionless ${ }^{2}$; in particular, symbol $B$ stands for a special combination of magnetic field amplitude, charge, mass, light velocity, and curvature radius

$$
B \Longleftrightarrow \frac{e}{m} \frac{\rho B}{c}, \quad t \Longleftrightarrow \frac{c t}{\rho}, \quad r \Longleftrightarrow \frac{r}{\rho}, \quad z \Longleftrightarrow \frac{z}{\rho} .
$$

\footnotetext{
${ }^{2}$ Bellow in the paper all relationships are written in that dimensionless form.
} 


\section{Particular solutions in Lobachevsky model}

Let us construct simple solutions when imposing the following constrain $r=r_{0}=$ const, equations (3.5) give

$$
\begin{aligned}
& V^{\phi}=-\frac{B}{\cosh r_{0}} \frac{1}{\cosh ^{2} z}, \\
& \frac{d}{d t}\left(-\frac{B}{\cosh r_{0}} \frac{1}{\cosh ^{2} z}\right)+2 \tanh z\left(-\frac{B}{\cosh r_{0}} \frac{1}{\cosh ^{2} z}\right) V^{z}=0, \\
& \frac{d V^{z}}{d t}=\left(\tanh ^{2} r_{0} B^{2}\right) \frac{\sinh z}{\cosh ^{3} z} .
\end{aligned}
$$

The second equation is an identity $0 \equiv 0$. With the notation

$$
\alpha=-B / \cosh r_{0}, \quad A=\left(\tanh ^{2} r_{0} B^{2}\right)>0,
$$

two remaining equations in (4.1) read

$$
\frac{d \phi}{d t}=\frac{\alpha}{\cosh ^{2} z}, \quad \frac{d V^{z}}{d t}=A \frac{\sinh z}{\cosh ^{3} z} .
$$

When $B>0$, the angular velocity $d \phi / d t<0$; and when $B<0$, the angular velocity $d \phi / d t>0$. Second equation in (4.2) means that there exists effective repulsion to both sides from the center $z=0$. One can resolve the second equation

$$
d\left(V^{z}\right)^{2}=A d\left(-\frac{1}{\cosh ^{2} z}\right) \quad \Longrightarrow \quad\left(\frac{d z}{d t}\right)^{2}=\epsilon-\frac{A}{\cosh ^{2} z} .
$$

Below we will see that the constant $\epsilon$ can be related to a squared velocity $V^{2} / c^{2}$ or differently to the integral of motion - the energy of the non-relativistic particle ${ }^{3}$. First, let $A \neq \epsilon$

$$
\pm \frac{d \sinh z}{\sqrt{\epsilon\left(1+\sinh ^{2} z\right)-A}}=d t
$$

Meaning of the signs \pm is evident: it corresponds to a motion along axis $z$ in opposite directions. Further we get

$$
\begin{aligned}
& \text { I. } \underline{\epsilon>A}, \quad z \in(-\infty,+\infty), \quad \pm \frac{1}{\sqrt{\epsilon}} \operatorname{arcsinh}\left(\sqrt{\frac{\epsilon}{\epsilon-A}} \sinh z\right)=t-t_{0} ; \\
& \text { II. } \underline{\epsilon<A}, \quad \sinh ^{2} z>\frac{A-\epsilon}{\epsilon}, \quad \pm \frac{1}{\sqrt{\epsilon}} \operatorname{arccosh}\left(\sqrt{\frac{\epsilon}{A-\epsilon}} \sinh z\right)=t-t_{0},
\end{aligned}
$$

or

$$
\begin{array}{ll}
\text { I. } \quad \underline{\epsilon>A}, \quad z \in(-\infty,+\infty), \quad \sinh z(t)= \pm \frac{\sqrt{\epsilon-A}}{\sqrt{\epsilon}} \sinh \sqrt{\epsilon}\left(t-t_{0}\right) \\
\text { II. } \quad \underline{\epsilon<A}, \quad \sinh ^{2} z>\frac{A-\epsilon}{\epsilon}, \quad \sinh z(t)= \pm \frac{\sqrt{A-\epsilon}}{\sqrt{\epsilon}} \cosh \sqrt{\epsilon}\left(t-t_{0}\right) .
\end{array}
$$

For motions of the type I, trajectories run through $z=0$; for motions of the type II, the particle is repulsed from the center $z=0$ at the points $\sinh z_{0}= \pm \sqrt{A / \epsilon-1}$. Existence of

\footnotetext{
${ }^{3}$ Relationship (4.3) in the limit of flat space will read $(d z / d t)^{2}=\epsilon-A$ which means that $A$ corresponds to a transversal squared velocity $V_{\perp}^{2} / c^{2}$. Also, equation (4.3) points out that in Lobachevsky model the transversal motion should vanish (to be frozen) when $z \rightarrow \pm \infty$.
} 
these two different regimes of motion along the axis $z$ correlates with the mentioned effective repulsion along the axis $z$.

Now let us consider the case $\epsilon=A$

$$
\left(\frac{d z}{d t}\right)^{2}=\epsilon \tanh ^{2} z
$$

We immediately see a trivial solution

$$
z(t)=0 \Longrightarrow \phi(t)=\phi_{0}+\alpha t, \quad \alpha=-\frac{B}{\cosh r_{0}}
$$

it corresponds to rotation of the particle along the circle $r=r_{0}$ in the absence of any motion along the axis $z$. Also there are non-trivial solutions to equation (4.4)

$$
\frac{d \sinh z}{\sinh z}= \pm \sqrt{\epsilon} d t
$$

here we have two different ones depending on sign $(+)$ or $(-)$. Continuous solutions of (4.5) exist only for $z>0$ and $z<0$ with different and peculiar properties. In the case of sign $(+)$ we have

$$
\sinh z=\sinh z_{0} e^{+\sqrt{\epsilon} t}, \quad\left(t=0, z=z_{0} \neq 0\right)
$$

at any positive initial $z_{0}>0$ the particle goes to $+\infty$; and at any negative initial $z_{0}<0$ the particle goes to $-\infty$. In the case of sign $(-)$, we get quite different behavior

$$
\sinh z=\sinh z_{0} e^{-\sqrt{\epsilon} t}, \quad\left(t=0, z=z_{0} \neq 0\right)
$$

at any positive initial $z_{0}>0$ the particle moves to $z=0$ during infinite time $t$; at any negative initial $z_{0}<0$ the particle moves to $z=0$ during infinite time $t$.

Now we are to turn to the first equation in (4.2) and find $\phi(t)$

$$
\begin{array}{ll}
\epsilon>A, & \phi-\phi_{0}=\frac{\alpha}{\sqrt{A}} \operatorname{arccoth}\left(\sqrt{\frac{A}{\epsilon}} \tanh \sqrt{\epsilon} t\right), \\
\epsilon<A, \quad \phi-\phi_{0}=\frac{\alpha}{\sqrt{A}} \operatorname{arccoth}\left(\sqrt{\frac{\epsilon}{A}} \tanh \sqrt{\epsilon} t\right) .
\end{array}
$$

One may note that when $t \rightarrow+\infty$ we obtain a finite value for the rotation angle

$$
\begin{aligned}
& \epsilon>A,\left.\quad\left(\phi-\phi_{0}\right)\right|_{t \rightarrow \infty}=\frac{\alpha}{\sqrt{A}} \operatorname{arccoth} \sqrt{\frac{A}{\epsilon}}, \\
& \epsilon<A,\left.\quad\left(\phi-\phi_{0}\right)\right|_{t \rightarrow \infty}=\frac{\alpha}{\sqrt{A}} \operatorname{arccoth} \sqrt{\frac{\epsilon}{A}} .
\end{aligned}
$$

In the same manner one should find $\phi(t)$ in case (4.6)

$$
\begin{aligned}
& A=\epsilon, \quad \sinh z=\sinh z_{0} e^{+\sqrt{\epsilon} t}, \\
& \phi-\phi_{0}=\alpha \int \frac{d t}{\cosh ^{2} z}=\alpha \int \frac{d t}{1+\sinh ^{2} z_{0} e^{+2 \sqrt{\epsilon} t}}, \quad x=\sinh ^{2} z_{0} e^{+2 \sqrt{\epsilon} t}, \\
& \phi-\phi_{0}=\left.\frac{\alpha}{2 \sqrt{\epsilon}} \ln \frac{x}{x+1}\right|_{t=0} ^{t}=\frac{\alpha}{2 \sqrt{\epsilon}}\left[\ln \frac{\sinh ^{2} z_{0}}{\sinh ^{2} z_{0}+e^{-2 \sqrt{\epsilon} t}}-\ln \frac{\sinh ^{2} z_{0}}{\sinh ^{2} z_{0}+1}\right] ;
\end{aligned}
$$


so that

$$
\phi-\phi_{0}=\frac{\alpha}{2 \sqrt{\epsilon}}=\ln \frac{\sinh ^{2} z_{0}+1}{\sinh ^{2} z_{0}+e^{-2 \sqrt{\epsilon}}}, \quad z_{0} \neq 0 .
$$

Again a peculiarity in the limit $t \rightarrow+\infty$ may be noted

$$
t \rightarrow+\infty, \quad \phi-\phi_{0}=\frac{\alpha}{2 \sqrt{\epsilon}} \ln \frac{\sinh ^{2} z_{0}+1}{\sinh ^{2} z_{0}}, \quad z_{0} \neq 0 .
$$

And the function $\phi(t)$ in case (4.7)

$$
\begin{aligned}
& A=\epsilon, \quad \sinh z=\sinh z_{0} e^{-\sqrt{\epsilon} t}, \\
& \phi-\phi_{0}=\alpha \int \frac{d t}{\cosh ^{2} z}=\alpha \int \frac{d t}{1+\sinh ^{2} z_{0} e^{-2 \sqrt{\epsilon} t}}, \quad x=\sinh ^{2} z_{0} e^{-2 \sqrt{\epsilon} t}, \\
& \phi-\phi_{0}=-\left.\frac{\alpha}{2 \sqrt{\epsilon}} \ln \frac{x}{x+1}\right|_{t=0} ^{t}=-\frac{\alpha}{2 \sqrt{\epsilon}}\left[\ln \frac{\sinh ^{2} z_{0}}{\sinh ^{2} z_{0}+e^{+2 \sqrt{\epsilon} t}}-\ln \frac{\sinh ^{2} z_{0}}{\sinh ^{2} z_{0}+1}\right] \text {, }
\end{aligned}
$$

so that

$$
\begin{aligned}
& \phi-\phi_{0}=-\frac{\alpha}{2 \sqrt{\epsilon}} \ln \frac{\sinh ^{2} z_{0}+1}{\sinh ^{2} z_{0}+e^{+2 \sqrt{\epsilon}}}, \\
& z_{0} \neq 0, \quad t \rightarrow+\infty, \quad \phi-\phi_{0}=\frac{\alpha}{2 \sqrt{\epsilon}}(+\infty) .
\end{aligned}
$$

Before going farther, let us note that from equation (2.6) it follows the conservation of squared velocity (or the energy) in the presence of a magnetic field

$$
\epsilon=\cosh ^{2} z\left[\left(\frac{d r}{d t}\right)^{2}+\sinh ^{2} r\left(\frac{d \phi}{d t}\right)^{2}\right]+\left(\frac{d z}{d t}\right)^{2} .
$$

For trajectories with constant $r=r_{0}$, the energy looks simpler (which coincides with (4.3))

$$
\epsilon=\sinh ^{2} r_{0} \frac{B^{2}}{\cosh ^{2} r_{0}} \frac{1}{\cosh ^{2} z}+\left(\frac{d z}{d z}\right)^{2}=\frac{A}{\cosh ^{2} z}+\left(\frac{d z}{d z}\right)^{2} .
$$

The above elementary treatment seems not to be completely satisfactory because we cannot be sure that all possible motions of the particle in a magnetic field in the Lobachevsky space have been found. So we turn to the Lagrange formalism.

\section{Particle in a magnetic field and Lagrange formalism in $\boldsymbol{H}_{3}$}

Now, let us consider the problem using the Lagrange formalism [15]

$$
\begin{aligned}
L & =\frac{1}{2}\left(-g_{i k} V^{i} V^{k}\right)-g_{i k} A^{i} V^{k} \\
& =\frac{1}{2}\left(\cosh ^{2} z V^{r} V^{r}+\cosh ^{2} z \sinh ^{2} r V^{\phi} V^{\phi}+V^{z} V^{z}\right)+B(\cosh r-1) V^{\phi} .
\end{aligned}
$$

Euler-Lagrange equations read

$$
\begin{aligned}
& \frac{d}{d t} \cosh ^{2} z V^{r}=\cosh ^{2} z \sinh r \cosh r V^{\phi} V^{\phi}+B \sinh r V^{\phi}, \\
& \frac{d}{d t}\left[\cosh ^{2} z \sinh ^{2} r V^{\phi}+B(\cosh r-1)\right]=0,
\end{aligned}
$$




$$
\frac{d}{d t} V^{z}=\cosh z \sinh z\left(V^{r} V^{r}+\sinh ^{2} r V^{\phi} V^{\phi}\right)
$$

or

$$
\begin{aligned}
& \frac{d V^{r}}{d t}+2 \tanh z V^{r} V^{z}-\sinh r \cosh r V^{\phi} V^{\phi}=B \frac{\sinh r}{\cosh ^{2} z} V^{\phi}, \\
& \frac{d V^{\phi}}{d t}+2 \operatorname{coth} r V^{\phi} V^{r}+2 \tanh z V^{\phi} V^{z}=-B \frac{1}{\cosh ^{2} z \sinh r} V^{r}, \\
& \frac{d V^{z}}{d t}=\sinh z \cosh z\left(V^{r} V^{r}+\sinh ^{2} r V^{\phi} V^{\phi}\right),
\end{aligned}
$$

which coincide with equations (3.5). Second equation in (5.1) evidently determines a new conserved quantity

$$
I=\cosh ^{2} z \sinh ^{2} r V^{\phi}+B(\cosh r-1)=\text { const. }
$$

We will obtain more from Lagrange formalism, if we use three integrals of motion. Two of them are already known

$$
\begin{aligned}
& I=\cosh ^{2} z \sinh ^{2} r V^{\phi}+B(\cosh r-1), \\
& \epsilon=\cosh ^{2} z\left(V^{r} V^{r}+\sinh ^{2} r V^{\phi} V^{\phi}\right)+V^{z} V^{z} .
\end{aligned}
$$

Having remembered equation (4.3), it is easy to guess the third

$$
A=\cosh ^{2} z\left[\epsilon-\left(\frac{d z}{d t}\right)^{2}\right]=\cosh ^{4} z\left(V^{r} V^{r}+\sinh ^{2} r V^{\phi} V^{\phi}\right)
$$

To see that $A$ indeed conserves it suffices to rewrite $A$ as

$$
A=\left(\cosh ^{2} z V^{r}\right)^{2}+\frac{1}{\sinh ^{2} r}\left(\cosh ^{2} z \sinh ^{2} r V^{\phi}\right)^{2},
$$

and takes into account the first and second equations in (5.1)

$$
\begin{aligned}
& \frac{d}{d t}\left(\cosh ^{2} z V^{r}\right)=\cosh ^{2} z \sinh r \cosh r V^{\phi} V^{\phi}+B \sinh r V^{\phi}, \\
& \frac{d}{d t}\left(\cosh ^{2} z \sinh ^{2} r V^{\phi}\right)=-B \sinh r V^{r}=0
\end{aligned}
$$

then we arrive at $d A / d t=0$. With the help of three integrals of motion we can reduce the problem in its most general form (without any additional and simplifying assumptions) to calculating several integrals. Indeed, from (5.2) it follows

$$
\frac{d \phi}{d t}=\frac{1}{\cosh ^{2} z} \frac{I-B(\cosh r-1)}{\sinh ^{2} r} .
$$

Substituting it into (5.3) we get

$$
A=\cosh ^{4} z\left(\frac{d r}{d t}\right)^{2}+\frac{[I-B(\cosh r-1)]^{2}}{\sinh ^{2} r},
$$

therefore

$$
\frac{d r}{d t}= \pm \frac{1}{\cosh ^{2} z} \sqrt{A-\frac{[I-B(\cosh r-1)]^{2}}{\sinh ^{2} r}} .
$$


In turn, from (5.3) it follows

$$
\frac{d z}{d t}= \pm \frac{1}{\cosh z} \sqrt{\epsilon \cosh ^{2} z-A} .
$$

Dividing (5.5) by (5.6), we obtain

$$
\frac{\sinh r d r}{\sqrt{A \sinh ^{2} r-(I-B \cosh r+B)^{2}}}= \pm \frac{1}{\cosh z} \frac{d z}{\sqrt{\epsilon \cosh ^{2} z-A}}
$$

which represents trajectory equation in the form $d F(r, z)=0$. In turn, dividing (5.5) by (5.4), we get trajectory equation in the form $d F(r, \phi)=0$

$$
\frac{[I-B(\cosh r-1)] d r}{\sinh r \sqrt{A \sinh ^{2} r-[I-B(\cosh r-1)]^{2}}}=d \phi .
$$

Thus, the solution of the problem - particle in a magnetic field on the background of Lobachevsky space - reduces to the following integrals

$$
\begin{aligned}
& \frac{d \phi}{d t}=\frac{1}{\cosh ^{2} z} \frac{I-B(\cosh r-1)}{\sinh ^{2} r}, \\
& \frac{d r}{d t}= \pm \frac{1}{\cosh ^{2} z} \sqrt{A-\frac{[I-B(\cosh r-1)]^{2}}{\sinh ^{2} r}} \\
& \frac{d z}{d t}= \pm \frac{1}{\cosh z} \sqrt{\epsilon \cosh ^{2} z-A}, \\
& \frac{\sinh r d r}{\sqrt{A \sinh ^{2} r-(I-B \cosh r+B)^{2}}}= \pm \frac{1}{\cosh z} \frac{d z}{\sqrt{\epsilon \cosh ^{2} z-A}}, \\
& \pm \frac{[I-B(\cosh r-1)] d r}{\sinh r \sqrt{A \sinh ^{2} r-[I-B(\cosh r-1)]^{2}}}=d \phi .
\end{aligned}
$$

The last five relations are valid for all possible solutions of the problem under consideration. In particular, let us show that the restriction $r=r_{0}=$ const is compatible with equations (5.7)(5.11). Indeed they give

$$
\begin{aligned}
& \frac{d \phi}{d t}=\frac{\alpha}{\cosh ^{2} z}, \quad \frac{d z}{d t}= \pm \frac{1}{\cosh z} \sqrt{\epsilon \cosh ^{2} z-A}, \\
& \alpha=\frac{I-B\left(\cosh r_{0}-1\right)}{\sinh ^{2} r_{0}}, \quad A=\frac{\left[I-B\left(\cosh r_{0}-1\right)\right]^{2}}{\sinh ^{2} r_{0}} .
\end{aligned}
$$

It should be noted that in Section 3 we had other representations for $\alpha$ and $A$

$$
\alpha=-\frac{B}{\cosh r_{0}}, \quad A=\tanh ^{2} r_{0} B^{2} .
$$

However they are equivalent. Indeed, equating two expressions for $\alpha$

$$
\frac{I-B\left(\cosh r_{0}-1\right)}{\sinh ^{2} r_{0}}=-\frac{B}{\cosh r_{0}} \quad \Longrightarrow \quad I=B \frac{1-\cosh r_{0}}{\cosh r_{0}}
$$

and substituting this into $A$ in (5.12) we get

$$
A=\frac{\left[I-B\left(\cosh r_{0}-1\right)\right]^{2}}{\sinh ^{2} r_{0}}
$$




$$
=\frac{B^{2}}{\sinh ^{2} r_{0}}\left[\frac{\left(1-\cosh r_{0}\right)}{\cosh r_{0}}+\left(1-\cosh r_{0}\right)\right]^{2}=\left(\tanh ^{2} r_{0} B^{2}\right)
$$

which coincides with (5.13). It should be specially noted that from (5.14) we must conclude that to the case of the most simple motion $r_{0}=$ const, there corresponds the inequality

$$
\cosh r_{0}=\frac{1}{1+I / B}>1, \quad \text { that is } \quad \frac{B}{I+B}>1
$$

From (5.15) we conclude that the motion with $r=r_{0}$ is possible if

$$
B>0, \quad-B<I<0 ; \quad B<0, \quad 0<I<-B .
$$

\section{Possible solutions in $\boldsymbol{H}_{3}$, radial finite and infinite motions}

Now, with the use of general relationships (5.7)-(5.11), let us examine the general case without restriction $r=r_{0}$. Let us $A \neq \epsilon$.

First, as shown above equation (5.9) results in
I. $\quad \epsilon>A, \quad z \in(-\infty,+\infty), \quad \sinh z(t)= \pm \sqrt{1-\frac{A}{\epsilon}} \sinh \sqrt{\epsilon} t$
II. $\quad A>\epsilon, \quad \sinh ^{2} z>\frac{A}{\epsilon}-1, \quad \sinh z(t)= \pm \sqrt{\frac{A}{\epsilon}-1} \cosh \sqrt{\epsilon} t$.

Equation (5.8) can be rewritten as

$$
\pm \int \frac{d \cosh r}{\sqrt{A\left(\cosh ^{2} r-1\right)-(I+B-B \cosh r)^{2}}}=\int \frac{d t}{\cosh ^{2} z(t)} .
$$

Integral in the right-hand side is known - see (4.8)

$$
\begin{aligned}
& \text { I. } \quad \epsilon>A, \quad R=\int \frac{d t}{\cosh ^{2} z}=\frac{1}{\sqrt{A}} \operatorname{arccoth}\left(\sqrt{\frac{A}{\epsilon}} \tanh \sqrt{\epsilon} t\right), \\
& \text { II. } \quad \epsilon<A, \quad R=\int \frac{d t}{\cosh ^{2} z}=\frac{1}{\sqrt{A}} \operatorname{arccoth}\left(\sqrt{\frac{\epsilon}{A}} \tanh \sqrt{\epsilon} t\right) .
\end{aligned}
$$

For the integral in the left-hand side we get

$$
\begin{aligned}
& L=\int \frac{d x}{\sqrt{a x^{2}+b x+c}}=\int \frac{d x}{\sqrt{a\left(x+\frac{b}{2 a}\right)^{2}+\frac{b^{2}-4 a c}{-4 a}}}, \quad x=\cosh r, \\
& a=A-B^{2}, \quad b=2 B(I+B), \quad c=-A-(I+B)^{2}<0 .
\end{aligned}
$$

It should be noted the identity

$$
a+b+c=-I^{2} .
$$

Depending on the values of $a, b, c$ there may be realized solutions of different types. 


\subsection{Finite radial motions}

Let it be $a<0, B^{2}-A>0$. The roots $x_{1}$ and $x_{2}$ are

$$
\begin{aligned}
& x_{1}=\frac{b-\sqrt{b^{2}-4 a c}}{-2 a}, \quad x_{2}=\frac{b+\sqrt{b^{2}-4 a c}}{-2 a}, \\
& b^{2}-4 a c=4 A\left[(I+B)^{2}-\left(B^{2}-A\right)\right],
\end{aligned}
$$

and inequality $b^{2}-4 a c>0$ reduces to the following restriction $(I+B)^{2}-\left(B^{2}-A\right)>0$. In general, at $a<0$ we might expect several possibilities

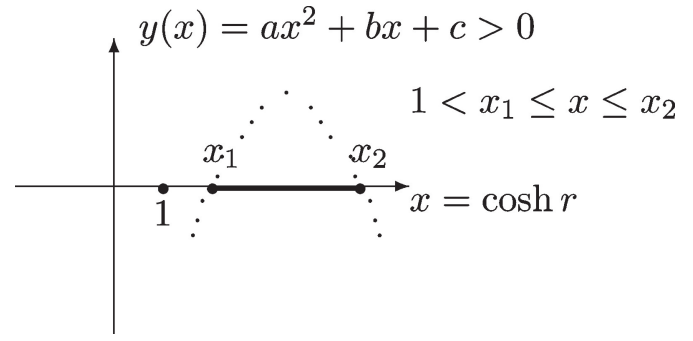

Fig. 1a. Finite motion.

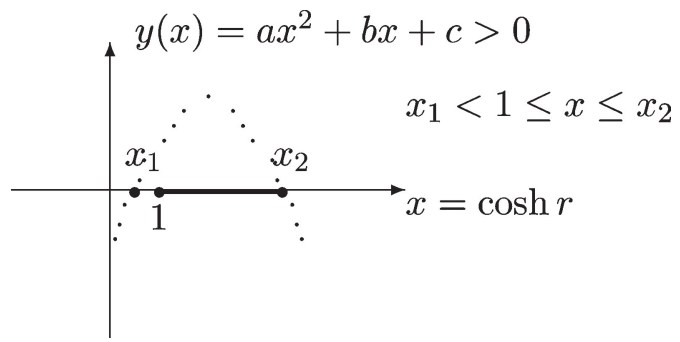

Fig. 1c. Finite motion.

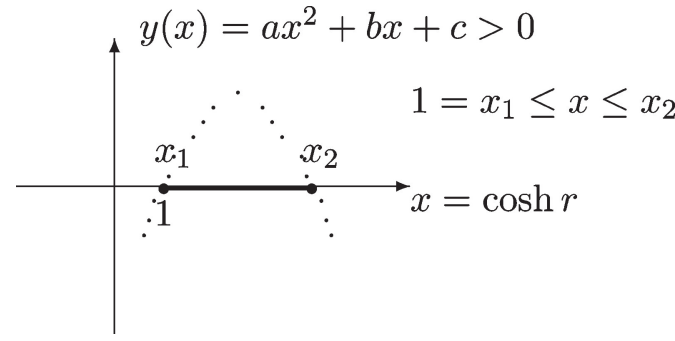

Fig. 1b. Finite motion.

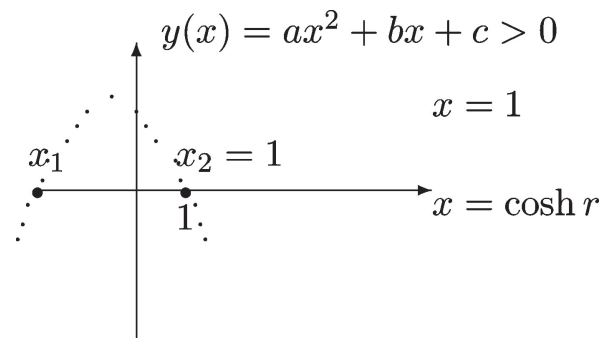

Fig. 1d. Very special state $r=0$.

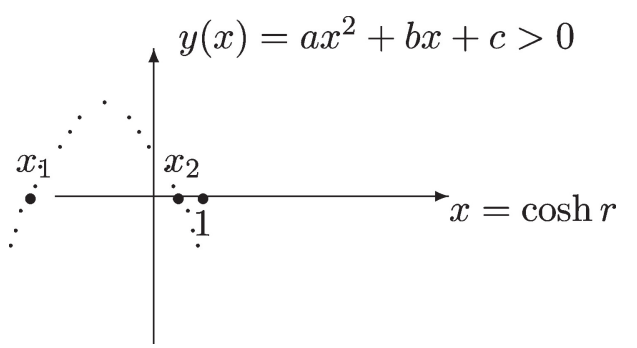

Fig. 1e. No physical solution.

Physically interesting cases, Figs. 1a-1c, can be characterized additionally by constrains on $a, b, c$

Fig. 1a $\quad b+2 a>0, \quad a+b+c<0 \quad \Longrightarrow$

$$
B I+A>0, \quad-I^{2}<0 \quad \text { (true inequality); }
$$

Fig. $1 \mathrm{~b} \quad b+2 a>0, \quad a+b+c=0 \quad \Longrightarrow \quad A>0, \quad I=0$

Fig. 1c $\quad a+b+c>0 \quad \Longrightarrow \quad-I^{2}>0 \quad$ (false statement).

Therefore, only Figs. 1a and 1b correspond to the physically possible solutions (with two turning points in radial variable)

Fig. 1a, $\quad B^{2}-A>0, \quad(I+B)^{2}-\left(B^{2}-A\right)>0, \quad B I+A>0, \quad I \neq 0$;

Fig. 1b, $\quad B^{2}-A>0, \quad A>0, \quad I=0$. 
Now let us turn to the integral (6.2) when (6.3) holds

$$
L=\int \frac{d x}{\sqrt{a\left(x+\frac{b}{2 a}\right)^{2}+\frac{b^{2}-4 a c}{-4 a}}}=\frac{1}{\sqrt{-a}} \arcsin \frac{-2 a x-b}{\sqrt{b^{2}-4 a c}}, \quad x_{1}<x<x_{2} .
$$

Therefore, equation (6.1) gives (let $\epsilon>A$ )

$$
\frac{1}{\sqrt{-a}} \arcsin \frac{-2 a \cosh r-b}{\sqrt{b^{2}-4 a c}}= \pm \frac{1}{\sqrt{A}} \operatorname{arccoth}\left(\sqrt{\frac{A}{\epsilon}} \tanh \sqrt{\epsilon} t\right),
$$

or

$$
\frac{-2 a \cosh r-b}{\sqrt{b^{2}-4 a c}}= \pm \sin \left[\frac{\sqrt{-a}}{\sqrt{A}} \operatorname{arccoth}\left(\sqrt{\frac{A}{\epsilon}} \tanh \sqrt{\epsilon} t\right)\right] .
$$

As expected, the variable $\cosh r$ runs within a finite segment

$$
x_{1}=\frac{b-\sqrt{b^{2}-4 a c}}{-2 a} \leq \cosh r \leq \frac{b+\sqrt{b^{2}-4 a c}}{-2 a}=x_{2},
$$

or (see Fig. 1a)

$$
\begin{aligned}
& B^{2}-A>0, \quad(I+B)^{2}-\left(B^{2}-A\right)>0, \quad B I+A>0, \\
& \frac{2 B(I+B)-\sqrt{4 A\left[(I+B)^{2}-\left(B^{2}-A\right)\right]}}{2\left(B^{2}-A\right)} \leq \cosh r, \\
& \cosh r \leq \frac{2 B(I+B)+\sqrt{4 A\left[(I+B)^{2}-\left(B^{2}-A\right)\right]}}{2\left(B^{2}-A\right)},
\end{aligned}
$$

which at $I=0$ takes a more simple form (see Fig. 1b)

$$
A<B^{2}, \quad A>0, \quad 1 \leq \cosh r \leq \frac{B^{2}+A}{B^{2}-A} .
$$

It should be noted that when

$$
b^{2}-4 a c=0 \quad \text { or equivalently } \quad B^{2}-A=(I+B)^{2}
$$

according to (6.4) the motion within the segment $\left[x_{1}, x_{2}\right]$ reduces to the motion with a fixed value $r_{0}$

$$
-2 a \cosh r_{0}-b=0 \quad \Longrightarrow \quad \cosh r_{0}=\frac{b}{-2 a}=\frac{2 B(I+B)}{2\left(B^{2}-A\right)}=\frac{B}{I+B},
$$

which coincides with the expression for $\cosh r_{0}$ given by (5.14).

\subsection{Infinite radial motions $(a>0)$}

One special case arises when $a=A-B^{2}=0$, indeed then we have

$$
\begin{aligned}
& L=\int \frac{d x}{\sqrt{b x+c}}=\frac{2}{b} \sqrt{b x+c}, \quad b>0, \quad x=\cosh r \geq-\frac{c}{b}, \\
& -\frac{c}{b}=\frac{B^{2}+(I+B)^{2}}{2 B(I+B)} \geq+1 \quad \Longrightarrow \quad B(I+B)>0,
\end{aligned}
$$


which corresponds to an infinite motion in radial variable (let $\epsilon>A$ )

$$
\frac{2}{\sqrt{b}} \sqrt{\cosh r+\frac{c}{b}}= \pm \frac{1}{\sqrt{B^{2}}} \operatorname{arccoth}\left(\sqrt{\frac{B^{2}}{\epsilon}} \tanh \sqrt{\epsilon} t\right) .
$$

Now let us examine other possibilities related to inequality $a>0$. When $a=A-B^{2}>0$, the roots $x_{1}, x_{2}$ are defined by

$$
x_{1}=\frac{-b-\sqrt{b^{2}-4 a c}}{2 a}, \quad x_{2}=\frac{-b+\sqrt{b^{2}-4 a c}}{2 a},
$$

and inequality $b^{2}-4 a c>0$ reduces to

$$
(I+B)^{2}+\left(A-B^{2}\right)>0 .
$$

In general, at the restrictions $a>0$ we might expect the following cases

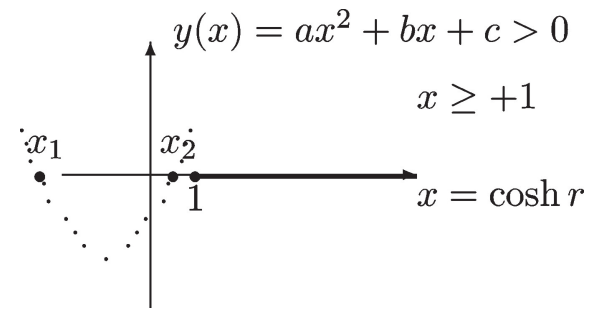

Fig. 2a. Infinite motion.

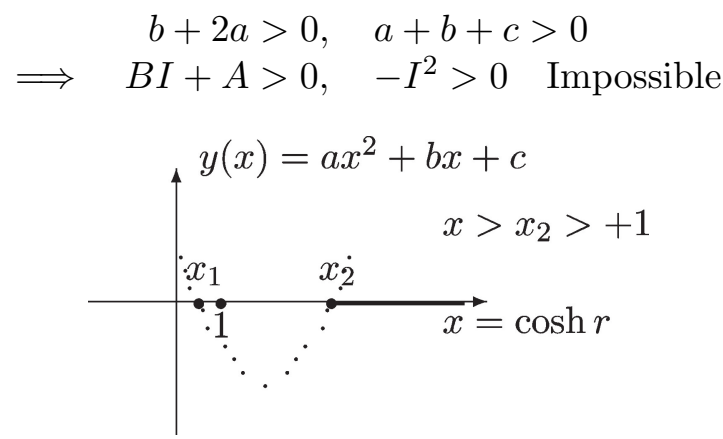

Fig. 2c. Infinite motion.

$$
\begin{gathered}
a+b+c<0 \\
\Longrightarrow \quad-I^{2}<0 \quad \text { Possible }
\end{gathered}
$$

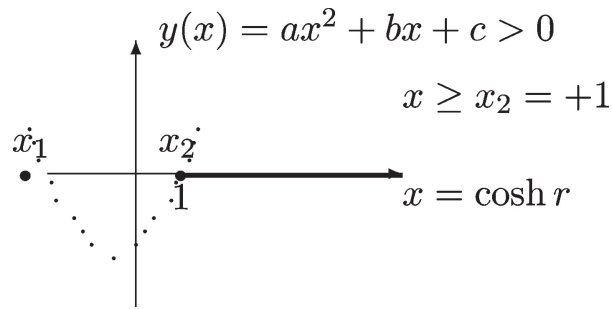

Fig. 2b. Infinite motion.

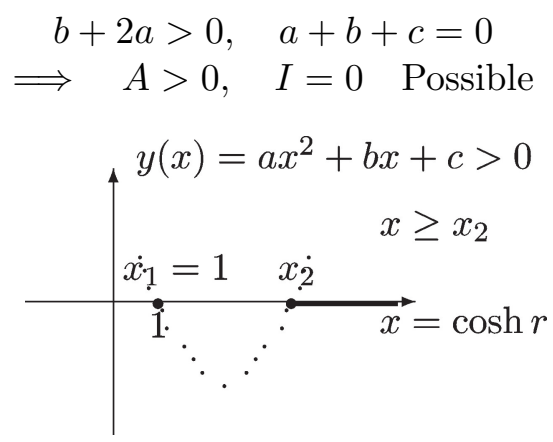

Fig. 2d. Infinite motion.

$$
\begin{gathered}
2 a+b<0, \quad a+b+c=0 \\
\Longrightarrow \quad A<0, \quad I=0 \quad \text { Impossible }
\end{gathered}
$$

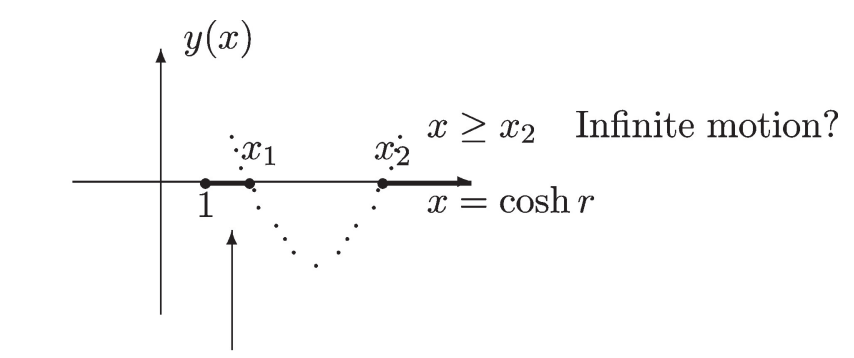

\section{$1 \leq x \leq x_{1} \quad$ Finite motion?}

Fig. 2e. Nonphysical case.

As concerns Fig. 2e, we should examine the following inequality

$$
x_{1}>1 \quad \Longrightarrow \quad-b>\sqrt{b^{2}-4 a c}+2 a \quad \Longrightarrow \quad(c-a)>+\sqrt{b^{2}-4 a c},
$$

which is impossible. 
Therefore, only Figs. 2b and 2c correspond to physically possible solutions (motions infinite in radial variable $r$ ). Integrating (6.2) reduces to the elementary calculation

$$
L=\int \frac{d x}{\sqrt{a\left(x+\frac{b}{2 a}\right)^{2}+\frac{b^{2}-4 a c}{-4 a}}}=\frac{1}{\sqrt{a}} \operatorname{arccosh} \frac{2 a x+b}{\sqrt{b^{2}-4 a c}} .
$$

Therefore, equation (6.1) gives (with $\epsilon>A$ )

$$
\frac{1}{\sqrt{a}} \operatorname{arccosh} \frac{2 a \cosh r+b}{\sqrt{b^{2}-4 a c}}= \pm \frac{1}{\sqrt{A}} \operatorname{arccoth}\left(\sqrt{\frac{A}{\epsilon}} \tanh \sqrt{\epsilon} t\right),
$$

or

$$
\frac{2 a \cosh r+b}{\sqrt{b^{2}-4 a c}}=\cosh \left(\frac{\sqrt{a}}{\sqrt{A}} \operatorname{arccoth}\left(\sqrt{\frac{A}{\epsilon}} \tanh \sqrt{\epsilon} t\right)\right),
$$

Evidently, equation (6.7) leads to (see Fig. 2c)

$$
\cosh r>\frac{-b+\sqrt{b^{2}-4 a c}}{2 a}, \quad b+2 a>0,
$$

or

$$
\cosh r>\frac{-2 B(I+B)+\sqrt{4 A\left[(I+B)^{2}+\left(A-B^{2}\right)\right]}}{2\left(A-B^{2}\right)}, \quad A+I B>0, \quad B^{2}<A
$$

from whence at $I=0$ it follows (see Fig. 2b) $\cosh r>+1, A>0$.

\section{$7 \quad$ Trajectory equation in the form $F(r, z)=0$, model $H_{3}$}

Now, let us consider the trajectory equation $F(r, z)=0$ according to (5.10).

$$
\int \frac{\sinh r d r}{\sqrt{A \sinh ^{2} r-(I-B \cosh r+B)^{2}}}= \pm \int \frac{1}{\cosh z} \frac{d z}{\sqrt{\epsilon \cosh ^{2} z-A}} .
$$

Its right-hand side gives

$$
\begin{aligned}
& \text { I. } \quad \epsilon>A, \quad z \in(-\infty,+\infty), \quad R= \pm \frac{1}{\sqrt{A}} \operatorname{arcsinh} \sqrt{\frac{A}{\epsilon-A}} \tanh z \\
& \text { II. } \quad \epsilon<A, \quad \sinh ^{2} z>\frac{A-\epsilon}{\epsilon}, \quad R= \pm \frac{1}{\sqrt{A}} \operatorname{arccosh} \sqrt{\frac{A}{A-\epsilon}} \tanh z .
\end{aligned}
$$

The left-hand side gives (two different possibilities depending on $\left(B^{2}, A\right)$ relation)

$$
\begin{aligned}
& \text { (a) }\left(B^{2}>A, \text { finite }\right) \quad L=\frac{1}{\sqrt{-a}} \arcsin \frac{-2 a \cosh r-b}{\sqrt{b^{2}-4 a c}}, \\
& \text { (b) }\left(B^{2} \leq A, \text { infinite }\right) \quad L=\frac{1}{\sqrt{a}} \operatorname{arccosh} \frac{2 a \cosh r+b}{\sqrt{b^{2}-4 a c}}
\end{aligned}
$$

Therefore, the trajectories $F(r, z)$ (5.10) have the form (four different cases)

$$
\epsilon>A, \quad B^{2}>A, \quad(I+B)^{2}>B^{2}-A, \quad z \in(-\infty,+\infty), \quad r \in\left(r_{1}, r_{2}\right),
$$




$$
\begin{aligned}
& \frac{1}{\sqrt{-a}} \arcsin \frac{-2 a \cosh r-b}{\sqrt{b^{2}-4 a c}}= \pm \frac{1}{\sqrt{A}} \operatorname{arcsinh}\left(\sqrt{\frac{A}{\epsilon-A}} \tanh z\right) ; \\
& \epsilon<A, \quad B^{2}>A, \quad(I+B)^{2}>B^{2}-A, \quad \sinh ^{2} z>\frac{A}{\epsilon}-1, \quad r \in\left(r_{1}, r_{2}\right), \\
& \frac{1}{\sqrt{-a}} \arcsin \frac{-2 a \cosh r-b}{\sqrt{b^{2}-4 a c}}= \pm \frac{1}{\sqrt{A}} \operatorname{arccosh}\left(\sqrt{\frac{A}{A-\epsilon}} \tanh z\right) ;
\end{aligned}
$$$$
\epsilon>A, \quad B^{2} \leq A, \quad z \in(-\infty,+\infty), \quad r \in\left(r_{1}, \infty\right)
$$$$
\frac{1}{\sqrt{a}} \operatorname{arccosh} \frac{2 a \cosh r+b}{\sqrt{b^{2}-4 a c}}= \pm \frac{1}{\sqrt{A}} \operatorname{arcsinh}\left(\sqrt{\frac{A}{\epsilon-A}} \tanh z\right)
$$$$
\epsilon<A, \quad B^{2} \leq A, \quad \sinh ^{2} z>\frac{A}{\epsilon}-1, \quad r \in\left(r_{1}, \infty\right),
$$$$
\frac{1}{\sqrt{a}} \operatorname{arccosh} \frac{2 a \cosh r+b}{\sqrt{b^{2}-4 a c}}= \pm \frac{1}{\sqrt{A}} \operatorname{arccosh}\left(\sqrt{\frac{A}{A-\epsilon}} \tanh z\right) \text {. }
$$

\section{Trajectory equation $F(r, \phi)=0$, the role of Lorentz $S O(3,1)$ transversal shifts in Lobachevsky space}

Now, let us consider the trajectory equation $F(r, \phi)(5.11)$

$$
\frac{[(I+B)-B \cosh r] d r}{\sinh r \sqrt{A \sinh ^{2} r-[(I+B)-B \cosh r]^{2}}}=d \phi .
$$

With the help of a new variable, the integral in the left-hand side reads

$$
u=\frac{(I+B) \cosh r-B}{\sinh r}, \quad L=-\int \frac{d u}{\sqrt{\left[\left(A-B^{2}\right)+(I+B)^{2}\right]-u^{2}}} .
$$

In connection to this integral we must require $\left[\left(A-B^{2}\right)+(I+B)^{2}\right]-u^{2}>0$, which can be transformed to the form

$$
\left(A-B^{2}\right) \cosh ^{2} r+2 B(I+B) \cosh r-A-(I+B)^{2}>0
$$

or (see Section 6) $x=\cosh r, a x^{2}+b x+c>0$; therefore, all analysis given in Section 6 is valid here as well. In particular, we should remember about necessary condition

$$
b^{2}-4 a c>0 \quad \Longrightarrow \quad(I+B)^{2}+\left(A-B^{2}\right)=C^{2}>0 .
$$

Integral (8.2) equals to

$$
L=\arccos \frac{u}{C}=\arccos \frac{(I+B) \cosh r-B}{\sinh r \sqrt{(I+B)^{2}+\left(A-B^{2}\right)}} ;
$$

correspondingly, equation (8.1) gives

$$
\arccos \frac{(I+B) \cosh r-B}{\sqrt{(I+B)^{2}+\left(A-B^{2}\right)} \sinh r}=\phi,
$$

or

$$
(I+B) \cosh r-\sqrt{(I+B)^{2}+\left(A-B^{2}\right)} \sinh r \cos \phi=B .
$$


This is the most general form of the trajectory equation $F(r, \phi)=0$. In particular, when $(I+B)^{2}+\left(A-B^{2}\right)=0$, equation (8.3) reads as relationship defining the motion of fixed radius $(I+B) \cosh r=B$, which coincides with (5.14).

One may assume that equation (8.3) when $B^{2}>A$ will describe a circle of fixed radius with shifted center in Lobachevsky space. As we see below this assumption is true. Indeed, let us introduce two coordinate systems in Lobachevsky space $H_{3}$

$$
\begin{aligned}
& u_{1}=\cosh z \sinh r \cos \phi, \quad u_{2}=\cosh z \sinh r \sin \phi, \quad u_{3}=\sinh z, \quad u_{0}=\cosh z \cosh r ; \\
& u_{1}^{\prime}=\cosh z^{\prime} \sinh r^{\prime} \cos \phi^{\prime}, \quad u_{2}^{\prime}=\cosh z^{\prime} \sinh r^{\prime} \sin \phi^{\prime}, \quad u_{3}^{\prime}=\sinh z^{\prime}, \quad u_{0}^{\prime}=\cosh z^{\prime} \cosh r^{\prime}
\end{aligned}
$$

related by a (Lorentz) shift in the plane (0-1)

$$
\left|\begin{array}{l}
u_{0}^{\prime} \\
u_{1}^{\prime} \\
u_{2}^{\prime} \\
u_{3}^{\prime}
\end{array}\right|=\left|\begin{array}{cccc}
\cosh \beta & \sinh \beta & 0 & 0 \\
\sinh \beta & \cosh \beta & 0 & 0 \\
0 & 0 & 1 & 0 \\
0 & 0 & 0 & 1
\end{array}\right|\left|\begin{array}{l}
u_{0} \\
u_{1} \\
u_{2} \\
u_{3}
\end{array}\right| .
$$

Equations (8.4) result in

$$
\begin{aligned}
& z^{\prime}=z, \quad \sinh r^{\prime} \sin \phi^{\prime}=\sinh r \sin \phi, \\
& \sinh r^{\prime} \cos \phi^{\prime}=\sinh \beta \cosh r+\cosh \beta \sinh r \cos \phi, \\
& \cosh r^{\prime}=\cosh \beta \cosh r+\sinh \beta \sinh r \cos \phi .
\end{aligned}
$$

In particular, $\beta$-shifted circle with fixed value $r^{\prime}=r_{0}^{\prime}$ will be described in coordinates $(r, \phi)$ by the following equation

$$
\cosh \beta \cosh r+\sinh \beta \sinh r \cos \phi=\cosh r_{0}^{\prime} .
$$

This equation should be compared with the above equation (8.3)

$$
(I+B) \cosh r-\sqrt{(I+B)^{2}-\left(A-B^{2}\right)} \sinh r \cos \phi=B,
$$

or (for simplicity, let $B$ and $I+B$ are both positive)

$$
\frac{(I+B)}{\sqrt{B^{2}-A}} \cosh r-\frac{\sqrt{(I+B)^{2}-\left(A-B^{2}\right)}}{\sqrt{B^{2}-A}} \sinh r \cos \phi=\frac{B}{\sqrt{B^{2}-A}} .
$$

Relations (8.6) and (8.7) coincide if the parameter $\beta$ and the radius of the shifted trajectory $r_{0}^{\prime}$ are defined according to

$$
\sinh \beta=-\frac{\sqrt{(I+B)^{2}-\left(A-B^{2}\right)}}{\sqrt{B^{2}-A}}, \quad \cosh r_{0}^{\prime}=\frac{B}{\sqrt{B^{2}-A}} .
$$

Let us turn back to the general equation for trajectories $F(r, \phi)=0$ according to (8.3)

$$
(I+B) \cosh r-\sqrt{(I+B)^{2}+\left(A-B^{2}\right)} \sinh r \cos \phi=B,
$$

and describe its behavior under (0-1)-shift (8.5)

$$
\begin{aligned}
& \sinh r \sin \phi=\sinh r^{\prime} \sin \phi^{\prime}, \\
& \sinh r \cos \phi=-\sinh \beta \cosh r^{\prime}+\cosh \beta \sinh r^{\prime} \cos \phi^{\prime}, \\
& \cosh r=\cosh \beta \cosh r^{\prime}-\sinh \beta \sinh r^{\prime} \cos \phi^{\prime} .
\end{aligned}
$$


Trajectory equation $F(r, \phi)=0$ translated to the coordinates $\left(r^{\prime}, \phi^{\prime}\right)$ looks

$$
\begin{aligned}
& (I+B)\left[\cosh \beta \cosh r^{\prime}-\sinh \beta \sinh r^{\prime} \cos \phi^{\prime}\right] \\
& \quad-\sqrt{(I+B)^{2}+\left(A-B^{2}\right)}\left[-\sinh \beta \cosh r^{\prime}+\cosh \beta \sinh r^{\prime} \cos \phi^{\prime}\right]=B,
\end{aligned}
$$

or

$$
\begin{aligned}
& {\left[\cosh \beta(I+B)+\sinh \beta \sqrt{(I+B)^{2}+\left(A-B^{2}\right)}\right] \cosh r^{\prime}} \\
& \quad-\left[\sinh \beta(I+B)+\cosh \beta \sqrt{(I+B)^{2}+\left(A-B^{2}\right)}\right] \sinh r^{\prime} \cos \phi^{\prime}=B .
\end{aligned}
$$

Comparing (8.8) and (8.9) we conclude that they are of the same form if two simple combinations of parameters are transformed by means of a Lorentz shift ${ }^{4}$

$$
\begin{aligned}
& I^{\prime}+B=\cosh \beta(I+B)+\sinh \beta \sqrt{(I+B)^{2}+\left(A-B^{2}\right)}, \\
& \sqrt{\left(I^{\prime}+B\right)^{2}+\left(A^{\prime}-B^{2}\right)}=\sinh \beta(I+B)+\cosh \beta \sqrt{(I+B)^{2}+\left(A-B^{2}\right)} .
\end{aligned}
$$

These Lorentz shifts leave invariant the following combination in the parametric space

$$
\operatorname{inv}=(I+B)^{2}-\left(\sqrt{(I+B)^{2}+\left(A-B^{2}\right)}\right)^{2}=B^{2}-A .
$$

This means that the Lorentz shifts vary in fact only the parameter $I$, whereas $A^{\prime}=A$. It makes sense to introduce new parameters $J, C$

$$
J=I+B, \quad C=+\sqrt{(I+B)^{2}+\left(A-B^{2}\right)}=\sqrt{I^{2}+2 I B+A}
$$

then equations (8.10) read

$$
J^{\prime}=J \cosh \beta+C \sinh \beta, \quad C^{\prime}=J \sinh \beta+C \cosh \beta
$$

and invariant form of the trajectory equation $F(r, \phi)=0$ can be presented as

$$
J \cosh r-C \sinh r \cos \phi=B,
$$

in any other shifted reference frame it must look as (this is a direct result from invariance property $B^{\prime}=B$ with respect to transversal Lorentz shifts)

$$
J^{\prime} \cosh r^{\prime}-C^{\prime} \sinh r^{\prime} \cos \phi^{\prime}=B .
$$

Correspondingly, the main invariant reads

$$
\text { inv }=J^{2}-C^{2}=J^{\prime 2}-C^{2}=B^{2}-A .
$$

Depending on the sign of this invariant one can reach the most simple description by means of appropriate shifts

1) $B^{2}-A>0$ (finite motion)

$$
J_{0}^{2}=B^{2}-A, \quad C_{0}=0, \quad \text { trajectory } \quad J_{0} \cosh r=B ;
$$

2) $B^{2}-A<0$ (infinite motion)

$$
J_{0}=0, \quad C_{0}^{2}=A-B^{2} \quad \text { trajectory } \quad-C_{0} \sinh r \cos \phi=B .
$$

One special case exists, see (6.5), (6.6).

3) $B^{2}=A$ (infinite motion)

$$
J=I+B, \quad C=I+B, \quad \text { trajectory } \quad \cosh r-\sinh r \cos \phi=\frac{B}{I+B} .
$$

By symmetry reasons, Lorentzian shifts of the type (0-2) will manifest themselves in the same manner.

\footnotetext{
${ }^{4}$ Below in Section 10 we will show that a magnetic field turns to be invariant under these Lorentz shifts, so $B^{\prime}=B$.
} 


\section{Lorentzian shifts and symmetry of a magnetic field in $\boldsymbol{H}_{3}$}

Let us turn again to a pair of coordinate systems in space $H_{3}$

$$
\begin{aligned}
& u_{1}=\cosh z \sinh r \cos \phi, \quad u_{2}=\cosh z \sinh r \sin \phi, \\
& u_{3}=\sinh z, \quad u_{0}=\cosh z \cosh r ; \\
& u_{1}^{\prime}=\cosh z^{\prime} \sinh r^{\prime} \cos \phi^{\prime}, \quad u_{2}^{\prime}=\cosh z^{\prime} \sinh r^{\prime} \sin \phi^{\prime}, \\
& u_{3}^{\prime}=\sinh z^{\prime}, \quad u_{0}^{\prime}=\cosh z^{\prime} \cosh r^{\prime},
\end{aligned}
$$

related by the shift $(0-1)$

$$
\left|\begin{array}{l}
u_{0}^{\prime} \\
u_{1}^{\prime} \\
u_{2}^{\prime} \\
u_{3}^{\prime}
\end{array}\right|=\left|\begin{array}{cccc}
\cosh \beta & \sinh \beta & 0 & 0 \\
\sinh \beta & \cosh \beta & 0 & 0 \\
0 & 0 & 1 & 0 \\
0 & 0 & 0 & 1
\end{array}\right|\left|\begin{array}{l}
u_{0} \\
u_{1} \\
u_{2} \\
u_{3}
\end{array}\right|,
$$

or in cylindric coordinates (direct and inverse formulas)

$$
\begin{aligned}
& z^{\prime}=z, \quad \sinh r^{\prime} \sin \phi^{\prime}=\sinh r \sin \phi, \\
& \sinh r^{\prime} \cos \phi^{\prime}=\sinh \beta \cosh r+\cosh \beta \sinh r \cos \phi, \\
& \cosh r^{\prime}=\cosh \beta \cosh r+\sinh \beta \sinh r \cos \phi ; \\
& z=z^{\prime}, \quad \sinh r \sin \phi=\sinh r^{\prime} \sin \phi^{\prime}, \\
& \sinh r \cos \phi=-\sinh \beta \cosh r^{\prime}+\cosh \beta \sinh r^{\prime} \cos \phi^{\prime}, \\
& \cosh r=\cosh \beta \cosh r^{\prime}-\sinh \beta \sinh r^{\prime} \cos \phi^{\prime} .
\end{aligned}
$$

With respect to that coordinate change $(r, \phi) \Longrightarrow\left(r^{\prime}, \phi^{\prime}\right)$, the uniform magnetic field transforms according to

$$
F_{\phi^{\prime} r^{\prime}}=\frac{\partial x^{\alpha}}{\partial \phi^{\prime}} \frac{\partial x^{\beta}}{\partial r^{\prime}} F_{\alpha \beta}=\left(\frac{\partial \phi}{\partial \phi^{\prime}} \frac{\partial r}{\partial r^{\prime}}-\frac{\partial r}{\partial \phi^{\prime}} \frac{\partial \phi}{\partial r^{\prime}}\right) F_{\phi r}, \quad F_{\phi r}=B \sinh r
$$

so that the magnetic field transforms by means of the Jacobian

$$
F_{\phi^{\prime} r^{\prime}}=J F_{\phi r}, \quad J=\left|\begin{array}{cc}
\frac{\partial r}{\partial r^{\prime}} & \frac{\partial r}{\partial \phi^{\prime}} \\
\frac{\partial \phi}{\partial r^{\prime}} & \frac{\partial \phi}{\partial \phi^{\prime}}
\end{array}\right|, \quad F_{\phi r}=B \sinh r .
$$

It is convenient to represent the coordinate transformation in the form

$$
\begin{aligned}
& \phi=\arctan \left(\frac{\sinh r^{\prime} \sin \phi^{\prime}}{-\sinh \beta \cosh r^{\prime}+\cosh \beta \sinh r^{\prime} \cos \phi^{\prime}}\right)=\arctan A, \\
& r=\operatorname{arccosh}\left(\cosh \beta \cosh r^{\prime}-\sinh \beta \sinh r^{\prime} \cos \phi^{\prime}\right)=\operatorname{arccosh} B
\end{aligned}
$$

correspondingly the Jacobian looks

$$
J=\frac{1}{\sqrt{B^{2}-1}} \frac{1}{1+A^{2}}\left(\frac{\partial B}{\partial r^{\prime}} \frac{\partial A}{\partial \phi^{\prime}}-\frac{\partial B}{\partial \phi^{\prime}} \frac{\partial A}{\partial r^{\prime}}\right) .
$$

Taking into account the identity

$$
\frac{1}{\sqrt{B^{2}-1}} \frac{1}{1+A^{2}}=\frac{1}{\sqrt{\cosh ^{2} r-1}} \frac{1}{1+\tan ^{2} \phi}=\frac{\cos ^{2} \phi}{\sinh r},
$$


and the formulas

$$
\begin{aligned}
\frac{\partial B}{\partial r^{\prime}} & =\frac{\partial}{\partial r^{\prime}}\left(\cosh \beta \cosh r^{\prime}-\sinh \beta \sinh r^{\prime} \cos \phi^{\prime}\right)=\cosh \beta \sinh r^{\prime}-\sinh \beta \cosh r^{\prime} \cos \phi^{\prime}, \\
\frac{\partial B}{\partial \phi^{\prime}} & =\frac{\partial}{\partial \phi^{\prime}}\left(\cosh \beta \cosh r^{\prime}-\sinh \beta \sinh r^{\prime} \cos \phi^{\prime}\right)=\sinh \beta \sinh r^{\prime} \sin \phi^{\prime}, \\
\frac{\partial A}{\partial \phi^{\prime}} & =\frac{\partial}{\partial \phi^{\prime}}\left(\frac{\sinh r^{\prime} \sin \phi^{\prime}}{-\sinh \beta \cosh r^{\prime}+\cosh \beta \sinh r^{\prime} \cos \phi^{\prime}}\right) \\
& =\frac{\sinh r^{\prime}\left(-\sinh \beta \cosh r^{\prime} \cos \phi^{\prime}+\cosh \beta \sinh r^{\prime}\right)}{\left(-\sinh \beta \cosh r^{\prime}+\cosh \beta \sinh r^{\prime} \cos \phi^{\prime}\right)^{2}} \\
& =\frac{\sinh r^{\prime}\left(-\sinh \beta \cosh r^{\prime} \cos \phi^{\prime}+\cosh \beta \sinh r^{\prime}\right)}{\sinh { }^{2} r \cos { }^{2} \phi}, \\
\frac{\partial A}{\partial r^{\prime}} & =\frac{\partial}{\partial r^{\prime}}\left(\frac{\sinh r^{\prime} \sin \phi^{\prime}}{-\sinh \beta \cosh r^{\prime}+\cosh \beta \sinh r^{\prime} \cos \phi^{\prime}}\right) \\
& =\frac{-\sinh \beta \sin \phi^{\prime}}{\left(-\sinh \beta \cosh r^{\prime}+\cosh \beta \sinh r^{\prime} \cos \phi^{\prime}\right)^{2}}=\frac{-\sinh \beta \sin \phi^{\prime}}{\sinh ^{2} r \cos ^{2} \phi},
\end{aligned}
$$

for the Jacobian we get

$$
\begin{aligned}
J= & \frac{\sinh r^{\prime}}{\sinh r} \frac{1}{\sinh ^{2} r}\left[\left(\cosh \beta \sinh r^{\prime}-\sinh \beta \cosh r^{\prime} \cos \phi^{\prime}\right)\right. \\
& \left.\times\left(-\sinh \beta \cosh r^{\prime} \cos \phi^{\prime}+\cosh \beta \sinh r^{\prime}\right)+\sinh ^{2} \beta \sin ^{2} \phi^{\prime}\right] .
\end{aligned}
$$

It is matter of simple calculation to verify that the denominator and numerator

$$
\begin{aligned}
& \sinh ^{2} r=\cosh ^{2} r-1=\left(\cosh \beta \cosh r^{\prime}-\sinh \beta \sinh r^{\prime} \cos \phi^{\prime}\right)^{2}-1 \\
& \quad=\cosh ^{2} \beta \cosh ^{2} r^{\prime}-2 \cosh \beta \sinh \beta \cosh r^{\prime} \sinh r^{\prime} \cos \phi^{\prime}+\sinh ^{2} \beta \sinh ^{2} r^{\prime} \cos ^{2} \phi^{\prime}-1, \\
& \left(\cosh \beta \sinh r^{\prime}-\sinh \beta \cosh r^{\prime} \cos \phi^{\prime}\right)\left(-\sinh \beta \cosh r^{\prime} \cos \phi^{\prime}+\cosh \beta \sinh r^{\prime}\right) \\
& \quad+\sinh ^{2} \beta \sin ^{2} \phi^{\prime}=-2 \cosh \beta \sinh \beta \cosh r^{\prime} \sinh r^{\prime} \cos \phi^{\prime} \\
& \quad+\cosh ^{2} \beta \sinh ^{2} r^{\prime}+\sinh ^{2} \beta \cosh ^{2} r^{\prime} \cos ^{2} \phi^{\prime}+\sinh ^{2} \beta \sin ^{2} \phi^{\prime}
\end{aligned}
$$

are equal to each other. Thus, the Jacobian of the shift (0-1) in hyperbolic space is

$$
J=\frac{\sinh r^{\prime}}{\sinh r}
$$

and therefore this shift leaves invariant the uniform magnetic field under consideration

$$
F_{\phi^{\prime} r^{\prime}}=J F_{\phi r}, \quad F_{\phi r}=B \sinh r, \quad F_{\phi^{\prime} r^{\prime}}=B \sinh r^{\prime} .
$$

By symmetry reason, we can conclude the same result for the shifts of the type (0-2). However, in that sense shifts of the type (0-3) behave differently. Indeed,

$$
\left|\begin{array}{l}
u_{0}^{\prime} \\
u_{1}^{\prime} \\
u_{2}^{\prime} \\
u_{3}^{\prime}
\end{array}\right|=\left|\begin{array}{cccc}
\cosh \beta & 0 & 0 & \sinh \beta \\
0 & 1 & 0 & 0 \\
0 & 0 & 1 & 0 \\
\sinh \beta & 0 & 0 & \cosh \beta
\end{array}\right|\left|\begin{array}{l}
u_{0} \\
u_{1} \\
u_{2} \\
u_{3}
\end{array}\right| .
$$

Equation (9.1) leads to relation between $(r, z)$ and $\left(r^{\prime}, z^{\prime}\right)$

$$
\begin{aligned}
& \cosh z^{\prime} \cosh r^{\prime}=\cosh \beta \cosh z \cosh r+\sinh \beta \sinh z, \\
& \sinh z^{\prime}=\sinh \beta \cosh z \cosh r+\cosh \beta \sinh z,
\end{aligned}
$$


$\cosh z^{\prime} \sinh r^{\prime}=\cosh z \sinh r, \quad \phi^{\prime}=\phi$.

Electromagnetic field is transformed according to

$$
F_{\alpha^{\prime} \beta^{\prime}}=\left(\frac{\partial \phi}{\partial x^{\prime \alpha}} \frac{\partial r}{\partial x^{\prime \beta}}-\frac{\partial r}{\partial x^{\prime \alpha}} \frac{\partial \phi}{\partial x^{\prime \beta}}\right) F_{\phi r} \quad \Longrightarrow \quad F_{\phi^{\prime} r^{\prime}}=\frac{\partial r}{\partial r^{\prime}} F_{\phi r}, \quad F_{\phi^{\prime} z^{\prime}}=\frac{\partial r}{\partial z^{\prime}} F_{\phi r}
$$

so the uniform magnetic field in the space $H_{3}$ is not invariant with respect to the shifts (0-3).

One may describe electromagnetic field in terms of 4-potential, and the rule to transform the field with respect to the shift $(0-1)$ looks

$$
A_{\phi}=-B(\cosh r-1) \quad \Longrightarrow \quad A_{\phi^{\prime}}^{\prime}=\frac{\partial \phi}{\partial \phi^{\prime}} A_{\phi}, \quad A_{r^{\prime}}^{\prime}=\frac{\partial \phi}{\partial r^{\prime}} A_{\phi} .
$$

In flat space, the shift (0-1) generates a definite gauge transformation

$$
\begin{aligned}
& \mathbf{A}(\mathbf{r})=\frac{1}{2} \mathbf{B} \times \mathbf{r}, \quad \mathbf{r}^{\prime}=\mathbf{r}+\mathbf{b}, \\
& \mathbf{A}^{\prime}\left(\mathbf{r}^{\prime}\right)=\frac{1}{2} \mathbf{B} \times \mathbf{r}^{\prime}-\frac{1}{2} \mathbf{B} \times \mathbf{b}=\frac{1}{2} \mathbf{B} \times \mathbf{r}^{\prime}+\nabla_{\mathbf{r}^{\prime}} \Lambda\left(x^{\prime}, y^{\prime}, z^{\prime}\right), \\
& \mathbf{B}=(0,0, B), \quad \mathbf{b}=(b, 0,0), \quad \Lambda\left(x^{\prime}, y^{\prime}, z^{\prime}\right)=-\frac{b B}{2} y^{\prime} .
\end{aligned}
$$

By analogy reason, one could expect something similar in the case of Lobachevsky space as well

$$
A_{\phi^{\prime}}^{\prime}=\frac{\partial \phi}{\partial \phi^{\prime}} A_{\phi}=-B\left(\cosh r^{\prime}-1\right)+\frac{\partial}{\partial \phi^{\prime}} \Lambda, \quad A_{r^{\prime}}^{\prime}=\frac{\partial \phi}{\partial r^{\prime}} A_{\phi}=\frac{\partial}{\partial r^{\prime}} \Lambda
$$

It is indeed so. Let us demonstrate this. Accounting for two formulas

$$
\begin{aligned}
\frac{\partial \phi}{\partial \phi^{\prime}} & =\frac{1}{1+A^{2}} \frac{\partial A}{\partial \phi^{\prime}}=\frac{\sinh r^{\prime}\left(-\sinh \beta \cosh r^{\prime} \cos \phi^{\prime}+\cosh \beta \sinh r^{\prime}\right)}{\sinh ^{2} r}, \\
\frac{\partial \phi}{\partial r^{\prime}} & =\frac{1}{1+A^{2}} \frac{\partial A}{\partial r^{\prime}}=\frac{-\sinh \beta \sin \phi^{\prime}}{\sinh ^{2} r},
\end{aligned}
$$

we conclude that the gauge function $\Lambda$ in (9.2) is defined by its partial derivatives in accordance with

$$
\begin{aligned}
\frac{\partial \Lambda}{\partial r^{\prime}} & =B \frac{\sinh \beta \sin \phi^{\prime}}{1+\cosh \beta \cosh r^{\prime}-\sinh \beta \sinh r^{\prime} \cos \phi^{\prime}}, \\
\frac{\partial \Lambda}{\partial \phi^{\prime}} & =B\left(\cosh r^{\prime}-1\right)-B \frac{\sinh r^{\prime}\left(\cosh \beta \sinh r^{\prime}-\sinh \beta \cosh r^{\prime} \cos \phi^{\prime}\right)}{1+\cosh \beta \cosh r^{\prime}-\sinh \beta \sinh r^{\prime} \cos \phi^{\prime}} \\
& =B \frac{\left(\cosh r^{\prime}-1\right)(1-\cosh \beta)+\sinh \beta \sinh r^{\prime} \cos \phi^{\prime}}{1+\cosh \beta \cosh r^{\prime}-\sinh \beta \sinh r^{\prime} \cos \phi^{\prime}} .
\end{aligned}
$$

The integrability condition $\partial^{2} \Lambda / \partial \phi^{\prime} \partial r^{\prime}=\partial^{2} \Lambda / \partial r^{\prime} \partial \phi^{\prime}$ in an explicit form looks

$$
\begin{aligned}
\frac{\partial}{\partial \phi^{\prime}} & \frac{\sinh \beta \sin \phi^{\prime}}{1+\cosh \beta \cosh r^{\prime}-\sinh \beta \sinh r^{\prime} \cos \phi^{\prime}} \\
& =\frac{\partial}{\partial r^{\prime}} \frac{\left(\cosh r^{\prime}-1\right)(1-\cosh \beta)+\sinh \beta \sinh r^{\prime} \cos \phi^{\prime}}{1+\cosh \beta \cosh r^{\prime}-\sinh \beta \sinh r^{\prime} \cos \phi^{\prime}} .
\end{aligned}
$$

It is the matter of simple direct calculation to verify it. Now we are to find an explicit form of the gauge function $\Lambda$. For better understanding, it is helpful first to consider a similar problem in flat space

$$
\text { space } E_{3}, \quad \frac{\partial \Lambda}{\partial r^{\prime}}=-B \frac{\alpha \sin \phi^{\prime}}{2}, \quad \frac{\partial \Lambda}{\partial \phi^{\prime}}=-B \frac{\alpha r^{\prime} \cos \phi^{\prime}}{2} .
$$


Integrating the first equation we obtain

$$
\Lambda=-\frac{B \alpha}{2} \sin \phi^{\prime} r^{\prime}+\lambda\left(\phi^{\prime}\right)
$$

and further from the second equation we derive

$$
-\frac{B \alpha}{2} \cos \phi^{\prime} r^{\prime}+\frac{d}{d \phi} \lambda\left(\phi^{\prime}\right)=-B \frac{\alpha r^{\prime} \cos \phi^{\prime}}{2} \quad \Longrightarrow \quad \lambda\left(\phi^{\prime}\right)=\lambda .
$$

Therefore, the gauge function is

$$
\Lambda\left(r^{\prime}, \phi^{\prime}\right)=-\frac{B \alpha}{2} \sin \phi^{\prime} r^{\prime}+\lambda=-\frac{B \alpha}{2} y^{\prime}+\lambda .
$$

The similar problem in space $H_{3}$ should be considered by the same scheme. Let us integrate the first equation in (9.3)

$$
\Lambda=B \sinh \beta \sin \phi^{\prime} \int \frac{d r^{\prime}}{1+\cosh \beta \cosh r^{\prime}-\sinh \beta \sinh r^{\prime} \cos \phi^{\prime}}+\lambda\left(\phi^{\prime}\right) .
$$

Introducing the notation $\cosh \beta=c, \sinh \beta=s, c^{2}-s^{2}=1$, and new variable

$$
\begin{aligned}
& \tanh \frac{r^{\prime}}{2}=y, \quad d r^{\prime}=2 \cosh ^{2} r^{\prime} d y \\
& \frac{d r^{\prime}}{1+\cosh \beta \cosh r^{\prime}-\sinh \beta \sinh r^{\prime} \cos \phi^{\prime}} \\
& \quad=\frac{2 \cosh ^{2} r^{\prime} d y}{1+c\left(\cosh ^{2} \frac{r^{\prime}}{2}+\sinh ^{2} \frac{r^{\prime}}{2}\right)-s 2 \sinh \frac{r^{\prime}}{2} \cosh \frac{r^{\prime}}{2} \cos \phi^{\prime}}=\frac{2 d y}{y^{2}(c-1)-2 y s \cos \phi^{\prime}+c+1},
\end{aligned}
$$

for $\Lambda$ we get

$$
\Lambda=\lambda\left(\phi^{\prime}\right)+2 B \arctan \frac{(c-1) y-s \cos \phi^{\prime}}{s \sin \phi^{\prime}},
$$

from whence it follows

$$
\Lambda=\lambda\left(\phi^{\prime}\right)+2 B \arctan \frac{(c-1)\left(\cosh r^{\prime}-1\right)-s \sinh r^{\prime} \cos \phi^{\prime}}{s \sinh r^{\prime} \sin \phi^{\prime}} .
$$

Now we are to calculate

$$
\begin{aligned}
\frac{\partial \Lambda}{\partial \phi^{\prime}} & =\frac{d \lambda}{d \phi^{\prime}}+2 B \frac{s^{2} \sinh ^{2} r^{\prime} \sin ^{2} \phi^{\prime}-\left[(c-1)\left(\cosh r^{\prime}-1\right)-s \sinh r^{\prime} \cos \phi^{\prime}\right] s \sinh r^{\prime} \cos \phi^{\prime}}{s^{2} \sinh ^{2} r^{\prime} \sin ^{2} \phi^{\prime}+\left[(c-1)\left(\cosh r^{\prime}-1\right)-s \sinh r^{\prime} \cos \phi^{\prime}\right]^{2}} \\
& =\frac{d \lambda}{d \phi^{\prime}}+2 B \frac{(c+1)\left(\cosh r^{\prime}+1\right)-s \sinh r^{\prime} \cos \phi^{\prime}}{(c+1)\left(\cosh r^{\prime}+1\right)+(c-1)\left(\cosh r^{\prime}-1\right)-2 s \sinh r^{\prime} \cos \phi^{\prime}}
\end{aligned}
$$

and finally

$$
\frac{\partial \Lambda}{\partial \phi^{\prime}}=\frac{d \lambda}{d \phi^{\prime}}+B \frac{\left(\cosh r^{\prime}+1\right)(c+1)-s \sinh r^{\prime} \cos \phi^{\prime}}{c \cosh r^{\prime}+1-s \sinh r^{\prime} \cos \phi^{\prime}} .
$$

After substituting into the second equation in (9.3) the expression for $\Lambda$ gives

$$
\frac{d \lambda}{d \phi^{\prime}}=B \frac{\left(\cosh r^{\prime}-1\right)(-c+1)+s \sinh r^{\prime} \cos \phi^{\prime}}{1+c \cosh r^{\prime}-s \sinh r^{\prime} \cos \phi^{\prime}}
$$




$$
+B \frac{\left(\cosh r^{\prime}+1\right)(-c-1)+s \sinh r^{\prime} \cos \phi}{1+c \cosh r^{\prime}-s \sinh r^{\prime} \cos \phi^{\prime}}=-2 B .
$$

Therefore,

$$
\lambda(\phi)=-2 B \phi+\lambda_{0}
$$

and the gauge function $\Lambda\left(r^{\prime}, \phi^{\prime}\right)$ is found as

$$
\Lambda\left(r^{\prime}, \phi^{\prime}\right)=+2 B \arctan \frac{(c-1)\left(\cosh r^{\prime}-1\right)-s \sinh r^{\prime} \cos \phi^{\prime}}{s \sinh r^{\prime} \sin \phi^{\prime}}-2 B \phi^{\prime}+\lambda_{0}
$$

remembering that $c=\cosh \beta, s=\sinh \beta$.

\section{Particle in a magnetic field, spherical Riemann model $S_{3}$}

In [18] under number XI we see the following system of coordinates in spherical space $S_{3}$

$$
\begin{aligned}
& d S^{2}=c^{2} d t^{2}-\rho^{2}\left[\cos ^{2} z\left(d r^{2}+\sin ^{2} r d \phi^{2}\right)+d z^{2}\right], \\
& z \in[-\pi / 2,+\pi / 2], \quad r \in[0,+\pi], \quad \phi \in[0,2 \pi], \\
& u^{1}=\cos z \sin r \cos \phi, \quad u^{2}=\cos z \sin r \sin \phi, \quad u^{3}=\sin z, \\
& u^{0}=\cos z \cos r, \quad\left(u^{0}\right)^{2}+\left(u^{1}\right)^{2}+\left(u^{2}\right)^{2}+\left(u^{3}\right)^{2}=1 .
\end{aligned}
$$

In these coordinates, let us introduce a magnetic field

$$
A_{\phi}=-2 B \sin ^{2} \frac{r}{2}=B(\cos r-1), \quad F_{\phi r}=\partial_{\phi} A_{r}-\partial_{r} A_{\phi}=B \sin r
$$

which satisfies the Maxwell equations in $S_{3}$

$$
\frac{1}{\cos ^{2} z \sin r} \frac{\partial}{\partial r} \cos ^{2} z \sin r\left(\frac{1}{\cos ^{4} z \sin ^{2} r}\right) B \sin r \equiv 0 .
$$

The Christoffel symbols read

$$
\begin{aligned}
\Gamma_{j k}^{r} & =\left|\begin{array}{ccc}
0 & 0 & -\tan z \\
0 & -\sin r \cos r & 0 \\
-\tan z & 0 & 0
\end{array}\right|, \quad \Gamma_{j k}^{\phi}=\left|\begin{array}{ccc}
0 & \cot r & 0 \\
\cot r & 0 & -\tan z \\
0 & -\tan z & 0
\end{array}\right|, \\
\Gamma_{j k}^{z} & =\left|\begin{array}{ccc}
\sin z \cos z & 0 & 0 \\
0 & \sin z \cos z \sin ^{2} r & 0 \\
0 & 0 & 0
\end{array}\right| .
\end{aligned}
$$

The non-relativistic equations of motion (2.9) in coordinates $(r, \phi, z)$ in a magnetic field look

$$
\begin{aligned}
& \frac{d V^{r}}{d t}-2 \tan z V^{r} V^{z}-\sin r \cos r V^{\phi} V^{\phi}=B \frac{\sin r}{\cos ^{2} z} V^{\phi}, \\
& \frac{d V^{\phi}}{d t}+2 \cot r V^{\phi} V^{r}-2 \tan z V^{\phi} V^{z}=-B \frac{1}{\cos ^{2} z \sin r} V^{r}, \\
& \frac{d V^{z}}{d t}+\sin z \cos z V^{r} V^{r}+\sin z \cos z \sin ^{2} r V^{\phi} V^{\phi}=0 .
\end{aligned}
$$

The last equation in (10.1) points that along the axis $z$ the effective attractive force acts to the center $z=0$. 


\section{Simplest solutions in spherical space}

Let us search for solutions with a fixed radius $r=r_{0}$; equations (10.1) give

$$
\begin{aligned}
& V^{\phi}=-\frac{B}{\cos r_{0}} \frac{1}{\cos ^{2} z}, \quad \frac{d}{d t}\left(-\frac{B}{\cos r_{0}} \frac{1}{\cos ^{2} z}\right)-2 \tan z\left(-\frac{B}{\cos r_{0}} \frac{1}{\cos ^{2} z}\right) V^{z}=0, \\
& \frac{d V^{z}}{d t}=-\left(\tan ^{2} r_{0} B^{2}\right) \frac{\sin z}{\cos ^{3} z}
\end{aligned}
$$

where the second equation is an identity $0 \equiv 0$. With notation $\alpha=-B / \cos r_{0}, A=B^{2} \tan ^{2} r_{0}$, the problem reduces to

$$
\frac{d \phi}{d t}=\frac{\alpha}{\cos ^{2} z}, \quad \frac{d V^{z}}{d t}=-A \frac{\sin z}{\cos ^{3} z} .
$$

The second equation gives

$$
d\left(V^{z}\right)^{2}=A d\left(-\frac{1}{\cos ^{2} z}\right) \quad \Longrightarrow \quad\left(\frac{d z}{d t}\right)^{2}=-\frac{A}{\cos ^{2} z}+\epsilon
$$

the constant $\epsilon$ will be related to the squared velocity. First, let $A \neq \epsilon$, then equation (11.2) gives (in contrast to Lobachevsky model, now only one possibility is realized: $\epsilon>A$ )

$$
\frac{d \sin z}{\sqrt{\epsilon\left(1-\sin ^{2} z\right)-A}}=d t
$$

Two different signs $( \pm)$ correspond to the motion of the particle in opposite directions along the axis $z$. The calculations below are evident (let $t_{0}=0$ )

$$
A \neq \epsilon, \quad \sin z(t)= \pm \frac{\sqrt{\epsilon-A}}{\sqrt{\epsilon}} \sin \sqrt{\epsilon} t .
$$

Let us examine a special case when $\epsilon=A$, equation (11.2) becomes

$$
\left(\frac{d z}{d t}\right)^{2}=-\epsilon \tan ^{2} z
$$

Equation (11.3) has only a trivial solution

$$
z(t)=0, \quad \text { so that } \quad \phi(t)=\phi_{0}+\alpha t, \quad \alpha=-\frac{B}{\cosh r_{0}},
$$

it corresponds to the rotation with constant angular velocity around the circle $r=r_{0}$ in the absence of any motion along the axis $z$.

Now we are to turn to the first equation in (11.1) and find $\phi(t)$

$$
A \neq \epsilon, \quad \phi=\alpha \int \frac{d t}{\cos ^{2} z}=\alpha \int \frac{d t}{\cos ^{2} \sqrt{\epsilon} t+\frac{A}{\epsilon} \sin ^{2} \sqrt{\epsilon} t},
$$

so that

$$
A \neq \epsilon, \quad \phi=\frac{\alpha}{\sqrt{A}} \arctan \left(\sqrt{\frac{A}{\epsilon}} \tan \sqrt{\epsilon} t\right) .
$$

Thus, we construct the following solution

$$
r=r_{0}=\text { const }, \quad A \neq \epsilon,
$$




$$
\sin z(t)= \pm \frac{\sqrt{\epsilon-A}}{\sqrt{\epsilon}} \sin \sqrt{\epsilon} t, \quad \phi=\frac{\alpha}{\sqrt{A}} \arctan \left(\sqrt{\frac{A}{\epsilon}} \tan \sqrt{\epsilon} t\right) .
$$

Distinctive feature of the motion is its periodicity and closeness of corresponding trajectories. The period $T$ is determined by the energy $\epsilon$

$$
T=\frac{\pi}{\sqrt{\epsilon}}, \quad \text { or in usual units } \quad T=\frac{\pi \rho}{V} .
$$

\section{Particle in a magnetic field and Lagrange formalism in $S_{3}$}

Let us consider the problem of a particle in a magnetic field in $S_{3}$ on the base of the Lagrange function

$$
L=\frac{1}{2}\left(\cos ^{2} z V^{r} V^{r}+\cos ^{2} z \sin ^{2} r V^{\phi} V^{\phi}+V^{z} V^{z}\right)-B(\cos r-1) V^{\phi} .
$$

Euler-Lagrange equations in the explicit form are

$$
\begin{aligned}
& \frac{d}{d t} \cos ^{2} z V^{r}=\cos ^{2} z \sin r \cos r V^{\phi} V^{\phi}+B \sin r V^{\phi}, \\
& \frac{d}{d t}\left[\cos ^{2} z \sin ^{2} r V^{\phi}-B(\cos r-1)\right]=0, \\
& \frac{d}{d t} V^{z}=-\cos z \sin z\left(V^{r} V^{r}+\sin ^{2} r V^{\phi} V^{\phi}\right),
\end{aligned}
$$

or differently

$$
\begin{aligned}
& \frac{d V^{r}}{d t}-2 \tan z V^{r} V^{z}-\sin r \cos r V^{\phi} V^{\phi}=B \frac{\sin r}{\cos ^{2} z} V^{\phi}, \\
& \frac{d}{d t}\left[\cos ^{2} z \sin ^{2} r V^{\phi}-B(\cos r-1)\right]=0, \\
& \frac{d}{d t} V^{z}=-\cos z \sin z\left(V^{r} V^{r}+\sin ^{2} r V^{\phi} V^{\phi}\right),
\end{aligned}
$$

which coincide with equations (10.1). Two integrals of motion in $S_{3}$ are known

$$
I=\cos ^{2} z \sin ^{2} r V^{\phi}-B(\cos r-1), \quad \epsilon=\cos ^{2} z\left(V^{r} V^{r}+\sin ^{2} r V^{\phi} V^{\phi}\right)+V^{z} V^{z},
$$

the third one can be constructed as follows

$$
A=\cos ^{2} z\left[\epsilon-\left(\frac{d z}{d t}\right)^{2}\right]=\cos ^{4} z\left(V^{r} V^{r}+\sin ^{2} r V^{\phi} V^{\phi}\right) .
$$

With the help of tree integrals of motion one can readily transform the problem under consideration to calculating several integrals

$$
\begin{aligned}
& \frac{d \phi}{d t}=\frac{1}{\cos ^{2} z} \frac{I+B(\cos r-1)}{\sin ^{2} r}, \\
& \frac{d r}{d t}= \pm \frac{1}{\cos ^{2} z} \sqrt{A-\frac{[I+B(\cos r-1)]^{2}}{\sin ^{2} r}} \\
& \frac{d z}{d t}= \pm \frac{1}{\cos z} \sqrt{\epsilon \cos ^{2} z-A}, \\
& \frac{\sin r d r}{\sqrt{A \sin ^{2} r-(I+B \cos r-B)^{2}}}= \pm \frac{1}{\cos z} \frac{d z}{\sqrt{\epsilon \cos ^{2} z-A}}
\end{aligned}
$$




$$
\frac{[I+B(\cos r-1)] d r}{\sin r \sqrt{A \sin ^{2} r-[I+B(\cos r-1)]^{2}}}=d \phi .
$$

For the most simple case when $r=r_{0}=$ const, these relations give

$$
\begin{aligned}
& \frac{d \phi}{d t}=\frac{\alpha}{\cosh ^{2} z}, \quad \frac{d z}{d t}= \pm \frac{1}{\cos z} \sqrt{\epsilon \cos ^{2} z-A}, \\
& \alpha=\frac{I+B\left(\cos r_{0}-1\right)}{\sin ^{2} r_{0}}, \quad A=\frac{\left[I+B\left(\cos r_{0}-1\right)\right]^{2}}{\sin ^{2} r_{0}} .
\end{aligned}
$$

In Section 11 for $\alpha$ and $A$ we had other expressions

$$
\alpha=-\frac{B}{\cos r_{0}}, \quad A=\left(\tan ^{2} r_{0} B^{2}\right)
$$

which were equivalent to the present ones. Indeed, from the identity $\alpha=\alpha$ we get

$$
\frac{I+B\left(\cos r_{0}-1\right)}{\sin ^{2} r_{0}}=-\frac{B}{\cos r_{0}} \quad \Longrightarrow \quad I=B \frac{\cos r_{0}-1}{\cos r_{0}},
$$

substituting it into (12.6) we arrive at

$$
A=\frac{\left[I+B\left(\cos r_{0}-1\right)\right]^{2}}{\sin ^{2} r_{0}}=\frac{B^{2}}{\sin ^{2} r_{0}}\left[\frac{\left(\cos r_{0}-1\right)}{\cos r_{0}}+\left(\cos r_{0}-1\right)\right]^{2}=\left(\tan ^{2} r_{0} B^{2}\right),
$$

which coincides with (12.7).

\section{All trajectories and $S O(4)$ symmetry of the space $S_{3}$}

Now, using general relations (12.1)-(12.5), let us examine the general case of possible solutions. First, let $A \neq \epsilon$. Relation (12.3) is integrated straightforwardly

$$
\sin z= \pm \frac{\sqrt{\epsilon-A}}{\sqrt{\epsilon}} \sin \sqrt{\epsilon} t
$$

Equation (12.2) reads

$$
\int \frac{\sin r d r}{\sqrt{A \sin ^{2} r-(I+B \cos r-B)^{2}}}= \pm \int \frac{d t}{\cos ^{2} z}
$$

The integral in the right-hand side is known, see (11.4),

$$
R= \pm \int \frac{d t}{\cos ^{2} z}= \pm \frac{1}{\sqrt{A}} \arctan \left(\sqrt{\frac{A}{\epsilon}} \tan \sqrt{\epsilon} t\right)
$$

The integral in the left-hand side is ${ }^{5}$

$$
\begin{aligned}
& L=\int \frac{\sin r d r}{\sqrt{A\left(1-\cos ^{2} r\right)-(I+B \cos r-B)^{2}}}=-\int \frac{d x}{\sqrt{a\left(x+\frac{b}{2 a}\right)^{2}+\frac{b^{2}-4 a c}{-4 a}}}, \\
& x=\cos r, \quad a=-A-B^{2}<0, \quad b=-2 B(I-B), \quad c=A-(I-B)^{2} .
\end{aligned}
$$

\footnotetext{
${ }^{5}$ In should be stressed that in the spherical model $S_{3}$ always $a<0$ which means that here only finite motions are possible.
} 


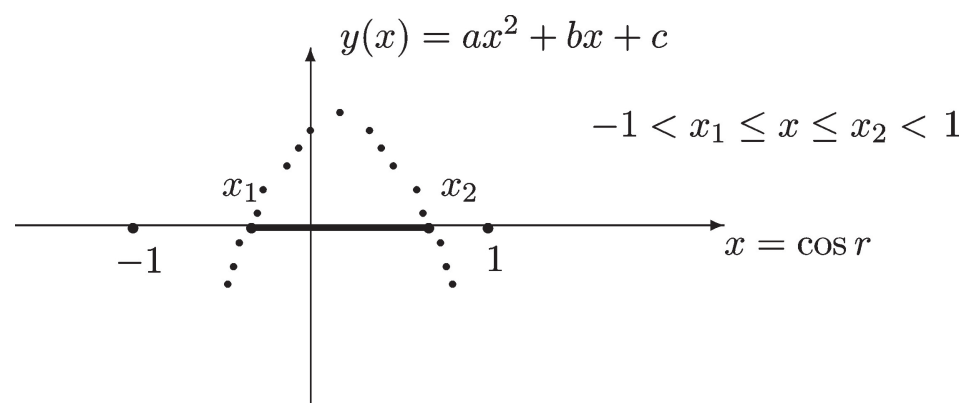

Fig. 3. Finite motion.

We may expect physical solutions when

$$
a<0, \quad b^{2}-4 a c>0, \quad-1 \leq \frac{b-\sqrt{b^{2}-4 a c}}{-2 a}=x_{1}<x<x_{2}=\frac{b+\sqrt{b^{2}-4 a c}}{-2 a} \leq 1 .
$$

First of all, we require $b^{2}-4 a c=4 A\left[A+B^{2}-(I-B)^{2}\right]>0$, that is

$$
b^{2}-4 a c>0 \quad \Longrightarrow \quad A+B^{2}>(I-B)^{2} \text {. }
$$

Also, we require

$$
-1 \leq x_{1}<x_{2} \leq+1 \quad \Longrightarrow \quad b-2 a \geq+\sqrt{b^{2}-4 a c}, \quad b+2 a \leq-\sqrt{b^{2}-4 a c} .
$$

From the first and second inequalities, it follows respectively:

$$
b-2 a \geq 0, \quad a+c-b \leq 0, \quad b+2 a \leq 0, \quad d a+c+b \leq 0 ;
$$

they are equivalent to

$$
\begin{array}{lll}
b-2 a \geq 0, & -(I-2 B)^{2} \leq 0, & -1 \leq x_{1}, \\
b+2 a \leq 0, & -I^{2} \leq 0, & x_{2} \leq+1 .
\end{array}
$$

Therefore, (13.3) will be satisfied if

$$
2 a \leq b \leq-2 a \quad \Longrightarrow \quad-\left(A+B^{2}\right) \leq-B(I-B) \leq\left(A+B^{2}\right) .
$$

Relationships (13.2) and (13.4) provide us with the constrains on parameters $(A, I, B)$ ensuring the existence of solutions in spherical space $S_{3}$. The integral (13.1) reduces to

$$
L=\int \frac{-d x}{\sqrt{a\left(x+\frac{b}{2 a}\right)^{2}+\frac{b^{2}-4 a c}{-4 a}}}=\frac{1}{\sqrt{-a}} \arcsin \frac{2 a \cos r+b}{\sqrt{b^{2}-4 a c}} .
$$

Therefore, equation (12.2) results in

$$
\frac{2 a \cos r+b}{\sqrt{b^{2}-4 a c}}=\sin \left[\frac{\sqrt{-a}}{\sqrt{A}} \operatorname{arccoth}\left(\sqrt{\frac{A}{\epsilon}} \tanh \sqrt{\epsilon} t\right)+\Lambda\right] .
$$

Now let us consider the trajectory equation (12.4) in the form $F(r, z)=0$. Its right-hand side after integration gives

$$
\pm \int \frac{1}{\cos z} \frac{d z}{\sqrt{\epsilon \cos ^{2} z-A}}= \pm \frac{1}{\sqrt{A}} \arcsin \sqrt{\frac{A}{\epsilon-A}} \tan z .
$$


Therefore, the trajectory equation (12.4) is

$$
\frac{1}{\sqrt{-a}} \arcsin \frac{2 a \cos r+b}{\sqrt{b^{2}-4 a c}}-\Lambda= \pm \frac{1}{\sqrt{A}} \arcsin \sqrt{\frac{A}{\epsilon-A}} \tan z .
$$

Now, let us consider equation (12.5) in the form $F(r, \phi)=0$

$$
\int \frac{[I+B(\cos r-1)] d r}{\sin r \sqrt{A \sin ^{2} r-[I+B(\cos r-1)]^{2}}}=\phi .
$$

Let us introduce a new variable

$$
u=\frac{(I-B) \cos r+B}{\sin r},
$$

the integral in (13.5) takes the form

$$
L=-\int \frac{d u}{\sqrt{\left(A+B^{2}\right)-(I-B)^{2}-u^{2}}}=\arccos \frac{(I-B) \cos r+B}{\sin r \sqrt{\left(A+B^{2}\right)-(I-B)^{2}}} .
$$

Therefore, the general trajectory equation $F(r, \phi)=0$ in the model $S_{3}$ looks

$$
(B-I) \cos r+\sqrt{\left(A+B^{2}\right)-(I-B)^{2}} \sin r \cos \phi=B .
$$

Let us consider the behavior of this equation with respect to (Euclidean) shifts (0-1) in spherical space. To this end, let us introduce two coordinate systems in $S_{3}$

$$
\begin{array}{llll}
u_{1}=\cos z \sin r \cos \phi, & u_{2}=\cos z \sin r \sin \phi, & u_{3}=\sin z, & u_{0}=\cos z \cos r, \\
u_{1}^{\prime}=\cos z^{\prime} \sin r^{\prime} \cos \phi^{\prime}, & u_{2}^{\prime}=\cos z^{\prime} \sin r^{\prime} \sin \phi^{\prime}, & u_{3}^{\prime}=\sin z^{\prime}, & u_{0}^{\prime}=\cos z^{\prime} \cos r^{\prime},
\end{array}
$$

related by the shift

$$
\left|\begin{array}{l}
u_{0}^{\prime} \\
u_{1}^{\prime} \\
u_{2}^{\prime} \\
u_{3}^{\prime}
\end{array}\right|=\left|\begin{array}{cccc}
\cos \alpha & \sin \alpha & 0 & 0 \\
-\sin \alpha & \cos \alpha & 0 & 0 \\
0 & 0 & 1 & 0 \\
0 & 0 & 0 & 1
\end{array}\right|\left|\begin{array}{l}
u_{0} \\
u_{1} \\
u_{2} \\
u_{3}
\end{array}\right| .
$$

Equation (13.7) gives the relation between two cylindric coordinate systems

$$
\begin{aligned}
& z^{\prime}=z, \quad \sin r^{\prime} \sin \phi^{\prime}=\sin r \sin \phi, \\
& \sin r^{\prime} \cos \phi^{\prime}=-\sin \alpha \cos r+\cos \alpha \sin r \cos \phi, \quad \cos r^{\prime}=\cos \alpha \cos r+\sin \alpha \sin r \cos \phi,
\end{aligned}
$$

and inverse ones

$$
\begin{aligned}
& z=z^{\prime}, \quad \sin r \sin \phi=\sin r^{\prime} \sin \phi^{\prime}, \\
& \sin r \cos \phi=\sin \alpha \cos r^{\prime}+\cos \alpha \sin r^{\prime} \cos \phi^{\prime}, \quad \cos r=\cos \alpha \cos r^{\prime}-\sin \alpha \sin r^{\prime} \cos \phi^{\prime} .
\end{aligned}
$$

Let us transform equation (13.6) to shifted coordinate $\left(r^{\prime}, \phi^{\prime}\right)$

$$
\begin{aligned}
& (B-I)\left[\cos \alpha \cos r^{\prime}-\sin \alpha \sin r^{\prime} \cos \phi^{\prime}\right] \\
& \quad+\sqrt{\left(A+B^{2}\right)+(I-B)^{2}}\left[\sin \alpha \cos r^{\prime}+\cos \alpha \sin r^{\prime} \cos \phi^{\prime}\right]=B .
\end{aligned}
$$

After elementary regrouping it reads

$$
\left[\cos \alpha(B-I)+\sin \alpha \sqrt{\left(A+B^{2}\right)-(I-B)^{2}}\right] \cos r^{\prime}
$$




$$
+\left[-\sin \alpha(B-I)+\cos \alpha \sqrt{\left(A+B^{2}\right)-(I-B)^{2}}\right] \sin r^{\prime} \cos \phi^{\prime}=B .
$$

Comparing (13.8) with (13.6), we see the invariance property of the trajectory equation if the parameters are transformed according to Euclidean rotation

$$
\begin{aligned}
& B^{\prime}-I^{\prime}=\cos \alpha(B-I)+\sin \alpha \sqrt{\left(A+B^{2}\right)-(I-B)^{2}} \\
& \sqrt{\left(A^{\prime}+B^{2}\right)-\left(I^{\prime}-B\right)^{2}}=-\sin \alpha(B-I)+\cos \alpha \sqrt{\left(A+B^{2}\right)-(I-B)^{2}},
\end{aligned}
$$

with notation $B-I=J, C=\sqrt{\left(A+B^{2}\right)-(I-B)^{2}}$, the trajectory equation has the following invariant form

$$
J \cos r+C \sin r \cos \phi=B \quad \Longrightarrow \quad J^{\prime} \cos r^{\prime}+C^{\prime} \sin r^{\prime} \cos \phi^{\prime}=B,
$$

with respect to Euclidean shifts $(0-1)$ in $S_{3}$ parameters $J, C$ transform according to

$$
J^{\prime}=J \cos \alpha+C \sin \alpha, \quad C^{\prime}=-J \sin \alpha+C \cos \alpha .
$$

This parametric shift generated by symmetry of the system leaves invariant the following (Euclidean) combination of two parameters

$$
\text { inv }=J^{2}+C^{2}=J^{\prime 2}+C^{2}=A+B^{2} \quad \Longrightarrow \quad A=A^{\prime}=\text { inv. }
$$

By a special choice of a shift one can translate the above equation (13.9) to more simple forms: for instance, to

$$
\begin{array}{llll}
J_{0}=\sqrt{A+B^{2}}, \quad C_{0}=0 & \Longrightarrow & J_{0} \cos r_{0}=B ; \\
J_{0}=0, \quad C_{0}=\sqrt{A+B^{2}} & \Longrightarrow & C_{0} \sin r \cos \phi=B .
\end{array}
$$

\section{Space shifts in space $S_{3}$ and gauge symmetry in $S_{3}$}

Let us introduce two cylindric coordinate systems

$$
\begin{aligned}
& u_{1}=\cos z \sin r \cos \phi, \quad u_{2}=\cos z \sin r \sin \phi, \\
& u_{3}=\sin z, \quad u_{0}=\cos z \cos r ; \\
& u_{1}^{\prime}=\cos z^{\prime} \sin r^{\prime} \cos \phi^{\prime}, \quad u_{2}^{\prime}=\cos z^{\prime} \sin r^{\prime} \sin \phi^{\prime}, \\
& u_{3}^{\prime}=\sin z^{\prime}, \quad u_{0}^{\prime}=\cos z^{\prime} \cos r^{\prime},
\end{aligned}
$$

related by the shift $(0-1)$

$$
\begin{aligned}
& \left|\begin{array}{l}
u_{0}^{\prime} \\
u_{1}^{\prime} \\
u_{2}^{\prime} \\
u_{3}^{\prime}
\end{array}\right|=\left|\begin{array}{cccc}
\cos \alpha & \sin \alpha & 0 & 0 \\
-\sin \alpha & \cos \alpha & 0 & 0 \\
0 & 0 & 1 & 0 \\
0 & 0 & 0 & 1
\end{array}\right|\left|\begin{array}{c}
u_{0} \\
u_{1} \\
u_{2} \\
u_{3}
\end{array}\right|, \quad z^{\prime}=z, \quad \sin r^{\prime} \sin \phi^{\prime}=\sin r \sin \phi, \\
& \sin r^{\prime} \cos \phi^{\prime}=-\sin \alpha \cos r+\cos \alpha \sin r \cos \phi, \quad \cos r^{\prime}=\cos \alpha \cos r+\sin \alpha \sin r \cos \phi .
\end{aligned}
$$

Under this change of variables, $(r, \phi) \Longrightarrow\left(r^{\prime}, \phi^{\prime}\right)$, the uniform magnetic field is transformed according to

$$
F_{\phi^{\prime} r^{\prime}}=\frac{\partial x^{\alpha}}{\partial \phi^{\prime}} \frac{\partial x^{\beta}}{\partial r^{\prime}} F_{\alpha \beta}=\left(\frac{\partial \phi}{\partial \phi^{\prime}} \frac{\partial r}{\partial r^{\prime}}-\frac{\partial r}{\partial \phi^{\prime}} \frac{\partial \phi}{\partial r^{\prime}}\right) F_{\phi r}, \quad F_{\phi r}=B \sinh r
$$


or in terms of the Jacobian

$$
F_{\phi^{\prime} r^{\prime}}=J F_{\phi r}, \quad J=\left|\begin{array}{cc}
\frac{\partial r}{\partial r^{\prime}} & \frac{\partial r}{\partial \phi^{\prime}} \\
\frac{\partial \phi}{\partial r^{\prime}} & \frac{\partial \phi}{\partial \phi^{\prime}}
\end{array}\right|, \quad F_{\phi r}=B \sin r .
$$

The coordinate transformation can be presented as

$$
\begin{aligned}
& \phi=\arctan \left(\frac{\sin r^{\prime} \sin \phi^{\prime}}{\sin \alpha \cos r^{\prime}+\cos \alpha \sin r^{\prime} \cos \phi^{\prime}}\right)=\arctan A, \\
& r=\arccos \left(\cos \alpha \cos r^{\prime}-\sin \alpha \sin r^{\prime} \cos \phi^{\prime}\right)=\arccos B .
\end{aligned}
$$

The Jacobian reads

$$
J=\frac{-1}{\sqrt{1-B^{2}}} \frac{1}{1+A^{2}}\left(\frac{\partial B}{\partial r^{\prime}} \frac{\partial A}{\partial \phi^{\prime}}-\frac{\partial B}{\partial \phi^{\prime}} \frac{\partial A}{\partial r^{\prime}}\right) .
$$

With the help of an identity

$$
\frac{-1}{\sqrt{1-B^{2}}} \frac{1}{1+A^{2}}=\frac{-1}{\sqrt{1-\cos ^{2} r}} \frac{1}{1+\tan ^{2} \phi}=-\frac{\cos ^{2} \phi}{\sin r},
$$

and the formulas

$$
\begin{aligned}
\frac{\partial B}{\partial r^{\prime}} & =\frac{\partial}{\partial r^{\prime}}\left(\cos \alpha \cos r^{\prime}-\sin \alpha \sin r^{\prime} \cos \phi^{\prime}\right)=-\cos \alpha \sin r^{\prime}-\sin \alpha \cos r^{\prime} \cos \phi^{\prime}, \\
\frac{\partial B}{\partial \phi^{\prime}} & =\frac{\partial}{\partial \phi^{\prime}}\left(\cos \alpha \cos r^{\prime}-\sin \alpha \sin r^{\prime} \cos \phi^{\prime}\right)=\sin \alpha \sin r^{\prime} \sin \phi^{\prime}, \\
\frac{\partial A}{\partial \phi^{\prime}} & =\frac{\partial}{\partial \phi^{\prime}}\left(\frac{\sin r^{\prime} \sin \phi^{\prime}}{\sin \alpha \cos r^{\prime}+\cos \alpha \sin r^{\prime} \cos \phi^{\prime}}\right) \\
& =\frac{\sin r^{\prime}\left(\sin \alpha \cos r^{\prime} \cos \phi^{\prime}+\cos \alpha \sin r^{\prime}\right)}{\left(\sin \alpha \cos r^{\prime}+\cos \alpha \sin r^{\prime} \cos \phi^{\prime}\right)^{2}}=\frac{\sin r^{\prime}\left(\sin \alpha \cos r^{\prime} \cos \phi^{\prime}+\cos \alpha \sin r^{\prime}\right)}{\sin ^{2} r \cos ^{2} \phi}, \\
\frac{\partial A}{\partial r^{\prime}} & =\frac{\partial}{\partial r^{\prime}}\left(\frac{\sin r^{\prime} \sin \phi^{\prime}}{\sin \alpha \cos r^{\prime}+\cos \alpha \sin r^{\prime} \cos \phi^{\prime}}\right) \\
& =\frac{\sin \alpha \sin \phi^{\prime}}{\left(\sin \alpha \cos r^{\prime}+\cos \alpha \sin r^{\prime} \cos \phi^{\prime}\right)^{2}}=\frac{\sin \alpha \sin \phi^{\prime}}{\sin ^{2} r \cos ^{2} \phi},
\end{aligned}
$$

for the Jacobian we get

$$
J=\frac{\sin r^{\prime}}{\sin r} \frac{\left(\cos \alpha \sin r^{\prime}+\sin \alpha \cos r^{\prime} \cos \phi^{\prime}\right)\left(\sin \alpha \cos r^{\prime} \cos \phi^{\prime}+\cos \alpha \sin r^{\prime}\right)+\sin ^{2} \alpha \sin ^{2} \phi^{\prime}}{\sin ^{2} r} .
$$

From whence it follows

$$
J=\frac{\sin r^{\prime}}{\sin r}
$$

therefore, a magnetic field is invariant under the shift (0-1) in space $S_{3}$

$$
F_{\phi^{\prime} r^{\prime}}=J F_{\phi r}, \quad F_{\phi r}=B \sin r, \quad F_{\phi^{\prime} r^{\prime}}=B \sin r^{\prime} .
$$

By symmetry reason, the same behavior of a magnetic field will take place for shifts of the type (0-2). However, for shifts of the type (0-3)

$$
\left|\begin{array}{c}
u_{0}^{\prime} \\
u_{1}^{\prime} \\
u_{2}^{\prime} \\
u_{3}^{\prime}
\end{array}\right|=\left|\begin{array}{cccc}
\cos \alpha & 0 & 0 & \sin \alpha \\
0 & 1 & 0 & 0 \\
0 & 0 & 1 & 0 \\
-\sin \alpha & 0 & 0 & \cos \alpha
\end{array}\right|\left|\begin{array}{c}
u_{0} \\
u_{1} \\
u_{2} \\
u_{3}
\end{array}\right|, \quad \cos z^{\prime} \cos r^{\prime}=\cos \alpha \cos z \cos r+\sin \alpha \sin z z,
$$




$$
\sin z^{\prime}=-\sin \alpha \cos z \cos r+\cos \alpha \sin z, \quad \cos z^{\prime} \sin r^{\prime}=\cos z \sin r, \quad \phi^{\prime}=\phi .
$$

electromagnetic field is transformed as

$$
F_{\alpha^{\prime} \beta^{\prime}}=\left(\frac{\partial \phi}{\partial x^{\prime \alpha}} \frac{\partial r}{\partial x^{\prime \beta}}-\frac{\partial r}{\partial x^{\prime \alpha}} \frac{\partial \phi}{\partial x^{\prime \beta}}\right) F_{\phi r} \quad \Longrightarrow \quad F_{\phi^{\prime} r^{\prime}}=\frac{\partial r}{\partial r^{\prime}} F_{\phi r}, \quad F_{\phi^{\prime} z^{\prime}}=\frac{\partial r}{\partial z^{\prime}} F_{\phi r}
$$

therefore a magnetic field is not invariant under these shifts $(0-3)$.

In terms of 4-potentials, the transformation rule reads

$$
A_{\phi}=B(\cos r-1) \quad \Longrightarrow \quad A_{\phi^{\prime}}^{\prime}=\frac{\partial \phi}{\partial \phi^{\prime}} A_{\phi}, \quad A_{r^{\prime}}^{\prime}=\frac{\partial \phi}{\partial r^{\prime}} A_{\phi}
$$

and we may expect

$$
A_{\phi^{\prime}}^{\prime}=\frac{\partial \phi}{\partial \phi^{\prime}} A_{\phi}=B\left(\cos r^{\prime}-1\right)+\frac{\partial}{\partial \phi^{\prime}} \Lambda, \quad A_{r^{\prime}}^{\prime}=\frac{\partial \phi}{\partial r^{\prime}} A_{\phi}=\frac{\partial}{\partial r^{\prime}} \Lambda .
$$

Accounting for two relations

$$
\begin{aligned}
\frac{\partial \phi}{\partial \phi^{\prime}} & =\frac{1}{1+A^{2}} \frac{\partial A}{\partial \phi^{\prime}}=\frac{\sin r^{\prime}\left(\sin \alpha \cos r^{\prime} \cos \phi^{\prime}+\cos \alpha \sin r^{\prime}\right)}{\sin ^{2} r}, \\
\frac{\partial \phi}{\partial r^{\prime}} & =\frac{1}{1+A^{2}} \frac{\partial A}{\partial r^{\prime}}=\frac{\sin \alpha \sin \phi^{\prime}}{\sin ^{2} r},
\end{aligned}
$$

for a 4-potential in shifted coordinates we have

$$
\begin{aligned}
& A_{\phi^{\prime}}^{\prime}=\frac{\sin r^{\prime}\left(\cos \alpha \sin r^{\prime}+\sin \alpha \cos r^{\prime} \cos \phi^{\prime}\right)}{\sin ^{2} r}[B(\cos r-1)], \\
& A_{r^{\prime}}^{\prime}=\frac{\sin \alpha \sin \phi^{\prime}}{\sin ^{2} r}[B(\cos r-1)],
\end{aligned}
$$

from whence it follows

$$
\begin{aligned}
& A_{\phi^{\prime}}^{\prime}=-B \frac{\sin r^{\prime}\left(\cos \alpha \sin r^{\prime}+\sin \alpha \cos r^{\prime} \cos \phi^{\prime}\right)}{1+\cos \alpha \cos r^{\prime}-\sin \alpha \sin r^{\prime} \cos \phi^{\prime}}, \\
& A_{r^{\prime}}^{\prime}=-B \frac{\sin \alpha \sin \phi^{\prime}}{1+\cos \alpha \cos r^{\prime}-\sin \alpha \sin r^{\prime} \cos \phi^{\prime}} .
\end{aligned}
$$

The gauge function $\Lambda$ in (14.1) is defined by its partial derivatives

$$
\begin{aligned}
\frac{\partial \Lambda}{\partial r^{\prime}} & =-B \frac{\sin \alpha \sin \phi^{\prime}}{1+\cos \alpha \cos r^{\prime}-\sin \alpha \sin r^{\prime} \cos \phi^{\prime}}, \\
\frac{\partial \Lambda}{\partial \phi^{\prime}} & =-B\left(\cos r^{\prime}-1\right)-B \frac{\sin r^{\prime}\left(\cos \alpha \sin r^{\prime}+\sin \alpha \cos r^{\prime} \cos \phi^{\prime}\right)}{1+\cos \alpha \cos r^{\prime}-\sin \alpha \sin r^{\prime} \cos \phi^{\prime}} \\
& =-B \frac{\left(\cos r^{\prime}-1\right)(1-\cos \alpha)+\sin \alpha \sin r^{\prime} \cos \phi^{\prime}}{1+\cos \alpha \cos r^{\prime}-\sin \alpha \sin r^{\prime} \cos \phi^{\prime}} .
\end{aligned}
$$

Integrability condition in the explicit form reads

$$
\begin{aligned}
\frac{\partial}{\partial \phi^{\prime}} & \left(-B \frac{\sin \alpha \sin \phi^{\prime}}{1+\cos \alpha \cos r^{\prime}-\sin \alpha \sin r^{\prime} \cos \phi^{\prime}}\right) \\
& =\frac{\partial}{\partial r^{\prime}}\left(-B \frac{\left(\cos r^{\prime}-1\right)(1-\cos \alpha)+\sin \alpha \sin r^{\prime} \cos \phi^{\prime}}{1+\cos \alpha \cos r^{\prime}-\sin \alpha \sin r^{\prime} \cos \phi^{\prime}}\right) .
\end{aligned}
$$


Now we are to find the gauge function $\Lambda$. Let us integrate the first equation in (14.2)

$$
\Lambda=-B \sin \alpha \sin \phi^{\prime} \int \frac{d r^{\prime}}{1+\cos \alpha \cos r^{\prime}-\sin \alpha \sin r^{\prime} \cos \phi^{\prime}}+\lambda\left(\phi^{\prime}\right)
$$

With the notation

$$
\begin{aligned}
& \cos \alpha=c, \quad \sin \alpha=s, \quad c^{2}+s^{2}=1, \quad \tan \frac{r^{\prime}}{2}=y, \quad d r^{\prime}=2 \cos ^{2} r^{\prime} d y, \\
& \frac{d r^{\prime}}{1+\cos \alpha \cos r^{\prime}-\sin \alpha \sin r^{\prime} \cos \phi^{\prime}} \\
& \quad=\frac{2 \cos ^{2} r^{\prime} d y}{1+c\left(\cos ^{2} \frac{r^{\prime}}{2}-\sin ^{2} \frac{r^{\prime}}{2}\right)-2 s \sin \frac{r^{\prime}}{2} \cos \frac{r^{\prime}}{2} \cos \phi^{\prime}}=\frac{2 d y}{y^{2}(1-c)-2 y s \cos \phi^{\prime}+c+1},
\end{aligned}
$$

for $\Lambda$ we obtain

$$
\Lambda=\lambda\left(\phi^{\prime}\right)-2 B \arctan \frac{(1-c)\left(1-\cos r^{\prime}\right)-s \sin r^{\prime} \cos \phi^{\prime}}{s \sin r^{\prime} \sin \phi^{\prime}} .
$$

Now, let us proceed further

$$
\begin{aligned}
\frac{\partial \Lambda}{\partial \phi^{\prime}} & =\frac{d \lambda}{d \phi^{\prime}}-2 B \frac{s^{2} \sin ^{2} r^{\prime} \sin ^{2} \phi^{\prime}-\left[(1-c)\left(1-\cos r^{\prime}\right)-s \sin r^{\prime} \cos \phi^{\prime}\right] s \sin r^{\prime} \cos \phi^{\prime}}{s^{2} \sin ^{2} r^{\prime} \sin ^{2} \phi^{\prime}+\left[(1-c)\left(1-\cos r^{\prime}\right)-s \sin r^{\prime} \cos \phi^{\prime}\right]^{2}} \\
& =\frac{d \lambda}{d \phi^{\prime}}-2 B \frac{(1+c)\left(1+\cos r^{\prime}\right)-s \sin r^{\prime} \cos \phi}{(1+c)\left(1+\cos r^{\prime}\right)+(1-c)\left(1-\cos r^{\prime}\right)-2 s \sin r^{\prime} \cos \phi^{\prime}},
\end{aligned}
$$

and finally

$$
\frac{\partial \Lambda}{\partial \phi^{\prime}}=\frac{d \lambda}{d \phi^{\prime}}-B \frac{(1+c)\left(1+\cos r^{\prime}\right)-s \sinh r^{\prime} \cos \phi}{1+c \cos r^{\prime}-s \sin r^{\prime} \cos \phi^{\prime}} .
$$

Substituting it into the second equation in (14.2) we get

$$
\frac{d \lambda}{d \phi^{\prime}}=-B \frac{\left(\cos r^{\prime}-1\right)(1-c)+s \sin r^{\prime} \cos \phi^{\prime}}{1+c \cos r^{\prime}-s \sin r^{\prime} \cos \phi^{\prime}}+B \frac{(1+c)\left(1+\cos r^{\prime}\right)-s \sinh r^{\prime} \cos \phi}{1+c \cos r^{\prime}-s \sin r^{\prime} \cos \phi^{\prime}}=2 B .
$$

Therefore,

$$
\lambda(\phi)=2 B \phi^{\prime}+\lambda_{0}
$$

Thus, the gauge function $\Lambda\left(r^{\prime}, \phi^{\prime}\right)$ is found (where $c=\cos \alpha, s=\sin \alpha$ )

$$
\Lambda\left(r^{\prime}, \phi^{\prime}\right)=-2 B \arctan \frac{(1-c)\left(1-\cos r^{\prime}\right)-s \sin r^{\prime} \cos \phi^{\prime}}{s \sin r^{\prime} \sin \phi^{\prime}}+2 B \phi^{\prime}+\lambda_{0} .
$$

\section{Extension to relativistic case}

Now let us briefly consider an extension to the relativistic problem - particle in a magnetic field in spaces $H_{3}$ and $S_{3}$. It suffices to discuss the hyperbolic case, then the relativistic equations have the form

$$
\frac{d}{d t}\left(\frac{1}{\sqrt{1-V^{2} / c^{2}}}\right)=0 \quad \Longrightarrow \quad \frac{1}{\sqrt{1-V^{2} / c^{2}}}=\frac{1}{\lambda}=\text { const }, \quad \lambda=\frac{m c^{2}}{E}<1
$$


and

$$
\begin{aligned}
& \frac{d}{d t} \frac{V^{1}}{\sqrt{1-V^{2} / c^{2}}}+\frac{1}{\sqrt{1-V^{2} / c^{2}}} \Gamma^{1}{ }_{j k} \frac{d x^{j}}{d t} \frac{d x^{k}}{d t}=-\left(V_{2} B^{3}-V_{3} B^{2}\right), \\
& \frac{d}{d t} \frac{V^{2}}{\sqrt{1-V^{2} / c^{2}}}+\frac{1}{\sqrt{1-V^{2} / c^{2}}} \Gamma^{2}{ }_{j k} \frac{d x^{j}}{d t} \frac{d x^{k}}{d t}=-\left(V_{3} B^{1}-V_{1} B^{3}\right), \\
& \frac{d}{d t} \frac{V^{3}}{\sqrt{1-V^{2} / c^{2}}}+\frac{1}{\sqrt{1-V^{2} / c^{2}}} \Gamma^{3}{ }_{j k} \frac{d x^{j}}{d t} \frac{d x^{k}}{d t}=-q\left(V_{1} B^{2}-V_{2} B^{1}\right)
\end{aligned}
$$

or in the explicit form

$$
\begin{aligned}
& \frac{d V^{r}}{d t}+2 \tanh z V^{r} V^{z}-\sinh r \cosh r V^{\phi} V^{\phi}=\lambda B \frac{\sinh r}{\cosh ^{2} z} V^{\phi}, \\
& \frac{d V^{\phi}}{d t}+2 \operatorname{coth} r V^{\phi} V^{r}+2 \tanh z V^{\phi} V^{z}=-\lambda B \frac{1}{\cosh ^{2} z \sinh r} V^{r}, \\
& \frac{d V^{z}}{d t}-\sinh z \cosh z V^{r} V^{r}-\sinh z \cosh z \sinh ^{2} r V^{\phi} V^{\phi}=0 .
\end{aligned}
$$

Therefore, all calculations performed for non-relativistic case are valid for relativistic case as well with the only change $B \Longrightarrow \lambda B$ and additional restriction $\epsilon<1$.

\section{Discussion}

Let us summarize the main results of the paper.

Motion of a classical particle in 3-dimensional Lobachevsky and Riemann spaces is studied in the presence of an external magnetic field which is analogous to a constant uniform magnetic field in Euclidean space. In both cases the equations of motion are solved exactly in special cylindrical coordinates. In Lobachevsky space there exist trajectories of two types: finite and infinite in radial variable, in Riemann space all motions are finite and periodical. The invariance of the uniform magnetic field in tensor description and gauge invariance of corresponding 4-potential description is demonstrated explicitly. The role of the symmetry is clarified in classification of all possible solutions given, based on the geometric symmetry group, $S O(3,1)$ and $S O(4)$ respectively.

Several additional points should be mentioned. Magnetic fields introduced in the models $H_{3}, S_{3}$ are not invariant under geometrical shift of the type (0-3), instead these geometrical transformations generate some additional electric fields. So, instead of four symmetry generators in Euclidean space, $\left(P_{1}, P_{2}, P_{3}, J_{z}\right)$, in curved space models we have only 3 generators for symmetries, $\left(J_{01}, J_{02}, J_{z}\right)$. This means that the magnetic fields under consideration in curved spaces are "less uniform" than in Euclidean space.

The choice of special coordinate systems is a matter of principal importance when exploring any problem, and our special choice of cylindrical coordinates is not accidental but turns to be decisive one. For instance, in Lobachevsky and Riemann models there exist other cylindrical coordinates in which Maxwell equations can be solved as well. In this case, electromagnetic potential is (in $H_{3}$ model)

$$
\begin{aligned}
& d S^{2}=d t^{2}-d r^{2}-d r^{2}-\sinh ^{2} d \phi^{2}-\cosh ^{2} d z^{2}, \quad A_{\phi}=\operatorname{const}[\ln (\cosh r)] \\
& u_{0}=\cosh r \cosh z, \quad u_{3}=\cosh r \sinh z, \quad u_{1}=\sinh r \cos \phi, \quad u_{0}=\sinh r \sin \phi,
\end{aligned}
$$

such a potential has a good limiting behavior at vanishing curvature limit and admits separating of variables, but it hardly could be brought to explicit analytical solutions when dealing with a particle behavior in this field. 
Up to now, the most attention in the literature was given to a Kepler problem (quantum mechanical and classical) in Lobachevsky and Riemann models and to the magnetic monopole problem. Corresponding electromagnetic potentials (in spherical coordinates) are

$$
H_{3}, \quad A_{0}=\frac{e}{\tanh r}, \quad A_{\phi}=g \cos \theta ; \quad S_{3}, \quad A_{0}=\frac{e}{\tan r}, \quad A_{\phi}=g \cos \theta .
$$

Magnetic field potentials used by us are

$$
H_{3}, \quad A_{\phi}=-B(\cosh r-1) ; \quad S_{3}, \quad A_{\phi}=B(\cos r-1) .
$$

They all provide us with solutions of the Maxwell equations. So all three potentials are equally correct. They are equally interesting as problems simple enough for their analytical treatment and as extensions of classical physical problems in flat space. At present time, we think, the third one is most interesting because one may expect new results on this field. For instance, as shown in [4] there exists special additional electric field that allows for solutions of corresponding Schrödinger equations in terms of hypergeometric functions, so one may expect the respective solutions of the classical equations in the presence of these additional electric fields as well. In this case one might expect to extend symmetry operations governing the structure of all solutions in presence of both uniform magnetic and electric fields.

\section{Acknowledgements}

Authors are grateful to participants of seminar of Laboratory of Theoretical Physics, National Academy of Sciences of Belarus for moral support and advice, also we are grateful to anonymous reviewers for stimulating discussion and criticism. This work was also supported by the Fund for Basic Researches of Belarus F09K-123. We wish to thank the Organizers of the VIII-th International Conference "Symmetry in Nonlinear Mathematical Physics" (June 21-27, 2009, Kyiv) for having given us the opportunity to talk on this subject as well as for local support.

\section{References}

[1] Avron J.E., Pnueli A., Landau Hamiltonians on symmetric spaces, in Ideas and Methods in Mathematical Analysis, Stochastics, and Applications (Oslo, 1988), Cambridge Univ. Press, Cambridge, 1992, 96-117.

[2] Bogush A.A., Red'kov V.M., Krylov G.G., Quantum particle in uniform magnetic field on the background of Lobachevsky space, Dokl. Nats. Akad. Nauk Belarusi 53 (2009), 45-51.

[3] Bogush A.A., Red'kov V.M., Krylov G.G., Quantum particle in uniform magnetic field on the background of spherical Riemann space, Vestsi Nats. Akad. Navuk Belarusi Ser. Fiz.-Mat. Navuk 2 (2009), 57-63.

[4] Bogush A.A., Red'kov V.M., Krylov G.G., Schrödinger particle in magnetic and electric fields in Lobachevsky and Riemann spaces, Nonlinear Phenom. Complex Syst. 11 (2008), 403-416.

[5] Cappelli A., Dunne G.V., Trugenberger C.A., Zemba G.R. Conformal symmetry and universal properties of quantum Hall states, Nuclear Phys. B. 398 (1993), 531-567, hep-th/9211071.

[6] Cappelli A., Trugenberger C.A., Zemba G.R., Infinite symmetry in the quantum Hall effect, Nuclear Phys. B 396 (1993), 465-490, hep-th/9206027.

[7] Cariñena J.F., Rañada M.F., Santander M., Central potentials on spaces of constant curvature: the Kepler problem on the two-dimensional sphere $S^{2}$ and the hyperbolic plane $H^{2}$, J. Math. Phys. 46 (2005), 052702, 25 pages, math-ph/0504016.

[8] Drukker N., Fiol B., Simón J., Gödel-type universes and the Landau problem, J. Cosmol. Astropart. Phys. 2004 (2004), no. 10, 012, 20 pages, hep-th/0309199.

[9] Dunne G.V., Hilbert space for charged particles in perpendicular magnetic fields, Ann. Physics 215 (1992), 233-263.

[10] Gadella M., Negro J., Pronko G.P., Santander M., Classical and quantum integrability in 3D system, J. Phys. A: Math. Theor. 41 (2008), 304030, 15 pages, arXiv:0711.4915. 
[11] Herranz J., Ballesteros A., Superintegrability on three-dimensional Riemannian and relativistic spaces of constant curvature, SIGMA 2 (2006), 010, 22 pages, math-ph/0512084.

[12] Klauder J.R., Onofri E., Landau levels and geometric quantization, Internat. J. Modern Phys. A 4 (1989), 3939-3949.

[13] Kudryashov V.V., Kurochkin Yu.A., Ovsiyuk E.M., Red'kov V.M., Motion caused by magnetic field in Lobachevsky space, in Proceedings of the 1st Zeldovich meeting "The Sun, the Stars, the Universe and General Relativity" (Minsk, April 20-23, 2009), Editors R. Ruffini and G. Vereshchagin, to appear.

[14] Landau L.D., Diamagnetismus der Metalle, Z. f. Physik 64 (1930), 629-637.

[15] Landau L.D., Lifshitz E.M., Theory of field, Nauka, Moscow, 1973.

[16] Landau L.D., Lifshitz E.M., Quantum mechanics, nonrelativistic theory, Nauka, Moscow, 1974.

[17] Negro J., del Olmo M.A., Rodríguez-Marco A., Landau quantum systems: an approach based on symmetry, J. Phys. A: Math. Gen. 35 (2002), 2283-2307, quant-ph/0110152.

[18] Olevsky M.N., Three-orthogonal coordinate systems in spaces of constant curvature, in which equation $\Delta_{2} U+\lambda U=0$ permits the full separation of variables, Mat. Sb. 27 (1950), 379-426.

[19] Onofri E., Landau levels on a torus, Internat. J. Theoret. Phys. 40 (2001), 537-549, quant-ph/0007055. 